\title{
MYCN mediates cysteine addiction and sensitizes to ferroptosis
}

Hamed Alborzinia ${ }^{1 * \# \pi}$, Andrés F. Flórez ${ }^{2 *}$, Sina Gogolin ${ }^{3 *}$, Lena M. Brückner ${ }^{3 *}$, Chunxuan Shao $^{2}$, Moritz Gartlgruber ${ }^{3}$, Michal Nadler-Holly ${ }^{4}$, Matthias Ziehm ${ }^{4}$, Franziska Paul ${ }^{5}$, Sebastian Steinhauser ${ }^{6}$, Emma Bell ${ }^{3}$, Marjan Shaikhkarami ${ }^{1}$, Sabine Hartlieb ${ }^{3}$, Daniel Dreidax $^{3}$, Elisa M. Hess ${ }^{3}$, Jochen Kreth ${ }^{3}$, Gernot Poschet ${ }^{7}$, Michael Büttner ${ }^{7}$, Barbara Nicke ${ }^{8}$, Carlo Stresemann ${ }^{9}$, Jan H. Reiling ${ }^{10}$, Matthias Fischer ${ }^{11}$, Ido Amit ${ }^{5}$, Matthias Selbach ${ }^{4}$, Carl Herrmann $^{6}$, Stefan Wölfl' ${ }^{1}$, Kai-Oliver Henrich ${ }^{3}$, Thomas Höfer ${ }^{2 \dagger}$ and Frank Westermann ${ }^{3 \# \dagger}$

*equal contribution; "Current address; ${ }^{\dagger}$ co-senior authors

${ }^{1}$ Institute of Pharmacy and Molecular Biotechnology, Heidelberg University, Heidelberg, Germany.

${ }^{2}$ Division of Theoretical Systems Biology, German Cancer Research Center (DKFZ), Heidelberg, Germany

${ }^{3}$ Division of Neuroblastoma Genomics, German Cancer Research Center (DKFZ) and Hopp-

Children's Comprehensive Cancer Center at the NCT (KiTZ), Heidelberg, Germany

${ }^{4}$ Proteome Dynamics, Max-Delbrück-Center for Molecular Medicine (MDC), Berlin, Germany

${ }^{5}$ Immunogenomics, Weizmann Institute of Science, Rehovot, Israel

${ }^{6}$ Division of Theoretical Bioinformatics (DKFZ), Heidelberg, Germany

${ }^{7}$ Metabolomics Core Technology Platform, University of Heidelberg, Heidelberg, Germany

${ }^{8}$ Target Discovery Technologies, Bayer AG, Berlin, Germany

${ }^{9}$ Drug Discovery, Pharmaceuticals Oncology I, Bayer AG, Berlin, Germany

${ }^{10}$ BioMed X Innovation Center, Heidelberg, Germany"

${ }^{11}$ Experimental Pediatric Oncology, Children's Hospital, and Center of Molecular Medicine (CMMC), Medical Faculty, University of Cologne, Cologne, Germany

"Corresponding authors:

Frank Westermann, M.D.

Division of Neuroblastoma Genomics,

German Cancer Research Center (DKFZ)

Im Neuenheimer Feld 280

D-69120 Heidelberg

Germany

Phone +49 6221423219

Fax +496221 423277

Email: f.westermann@dkfz.de

Hamed Alborzinia, Ph.D., D.V.M.

Institute of Pharmacy and Molecular Biotechnology (IPMB)

Heidelberg University

Im Neuenheimer Feld 364

D-69120 Heidelberg

Germany

Phone +49 6221544880

Fax +496221544884

Email: hamed.alborzy@gmail.com 


\section{Abstract}

Aberrant expression of MYC family members predicts poor clinical outcome in many human cancers. Oncogenic MYC profoundly alters metabolism and mediates an antioxidant response to maintain redox balance. Here we show that MYC induces massive lipid peroxidation upon depletion of cysteine, the rate-limiting amino acid for glutathione biosynthesis and sensitizes cells to ferroptosis, an oxidative, non-apoptotic and irondependent type of cell death. In MYCN-amplified childhood neuroblastoma, MYCN mediates resistance to ferroptosis by activating transsulfuration of methionine to cysteine. MYCN may contribute to spontaneous tumor regression in low-risk neuroblastomas by promoting ferroptosis in cells with epigenetically silenced cystathionine-beta-synthase, the rate-limiting enzyme for transsulfuration. We identified enzymes and antiporter proteins crucial to ferroptotic escape, providing multiple previously unknown sites that may be acted on therapeutically. 


\section{Introduction}

Many human cancers depend on aberrant expression of MYC transcription factor family members for unrestricted growth and proliferation, with high expression levels predicting poor clinical outcome ${ }^{1-4}$. How MYC-mediated metabolic adaptations ensure nutritional uptake and synthesis of cellular building blocks required for rapidly expanding cell populations remains poorly understood. Experimental evidence ${ }^{5-8}$ has long been interpreted as addiction of MYC-driven cancer cells to the amino acid glutamine, which when absent causes apoptosis. The finding that MYC can promote cell death as well as cell proliferation remains paradoxical in view of MYC oncogenic potential. Transgenic mouse models support MYC requirement for both tumor development and functions triggering massive cell death depending on tissue type and context ${ }^{5}$. Whether these cell death-promoting activities are irreversibly impaired by mutations in MYC-driven cancer cells or may be reactivated remains to be clarified.

Childhood neuroblastoma, an embryonic tumor derived from progenitors of the sympathetic nervous system, is a paradigmatic model for MYC-driven cancers ${ }^{6}$. MYCN amplification identifies a highly aggressive subtype associated with malignant progression and poor outcome despite intensive multimodal therapy ${ }^{7,8}$. In contrast, a relatively large fraction of low-risk neuroblastomas with elevated MYCN expressed from a normal MYCN locus, particularly those arising in children under 18 months of age, regress spontaneously even if the disease is metastatic ${ }^{9,10}$. While the forms of regulated cell death involved are unclear, genes required for apoptosis, such as caspase 8 (CASP8), are often epigenetically silenced and anti-apoptotic signals, such as expression of the MDM2 proto-oncogene, are active in primary high-risk and relapse neuroblastomas ${ }^{11-13}$.

\section{Results}

\section{Cystine deprivation induces ferroptosis}

We analyzed the interplay of oncogenic MYCN or MYC, referred to here as MYC $(N)$, activity with amino acid metabolism. Proliferation was slowed without inducing death (Extended Data Fig. 1a, b) by downregulating MYCN to a 'MYCN-low' state ( $65 \%$ reduction, Fig. 1a) in the MYCN-amplified IMR5/75 neuroblastoma cell model ${ }^{14}$, and intracellular pools of all amino acids were reduced (Fig. 1b), most prominently cysteine up to tenfold (Fig. 1c). Inhibiting MYCN binding to MYC associated factor X (MAX) with $10058-\mathrm{F} 4^{15}$ achieved similar results (Fig. 1b and Extended Data Fig. 1c). These experiments suggest that an oncogenic 'MYCNhigh' cellular background heavily utilizes cysteine. Systematically depleting individual amino acids from the growth medium confirmed the selective dependence of cells in the 'MYCNhigh' state on cystine (oxidized form of cysteine imported by cells and readily reduced to two 
cysteine molecules). Cystine deprivation caused cell death preferentially in 'MYCN-high' cells, and was more detrimental in this context than the previously described glutamine deprivation ${ }^{16-19}$ (Fig. 1d). Instituting a 'MYCN-low' cellular background inhibited cell death induced by cystine deprivation (Fig.1d). Moreover, inhibiting MYCN-MAX binding for MYCN function largely rescued cystine-deprived 'MYCN-high' cells from cell death (Fig. 1e). Overexpressing MYCN in a diploid MYCN background using the Tet21N neuroblastoma cell model $^{20}$ (Fig. 1a) further confirmed cystine addiction in cells with a 'MYCN-high' state (Fig. 1f). We stably integrated a regulable element capable of reducing MYC protein levels by $\sim 85 \%$ (Fig. 1a) in the MYC-amplified $\mathrm{NCl}-\mathrm{H} 23$ lung cancer cell line, and showed that cystine deprivation also caused cell death in the 'MYC-high' cellular background that could be largely prevented by MYC knockdown (Fig. 1g). These data suggest that survival of MYC(N)-driven tumor cells acutely depends on cystine availability, which maintains an adequate intracellular cysteine supply.

To investigate the form of regulated cell death induced by cystine deprivation, we used small molecule inhibitors. Inhibiting caspase proteases to prevent apoptosis, lysosomal function to inhibit autophagy, or RIPK1 to prevent necroptosis, did not abolish IMR5/75 cell death in the 'MYCN-high', cystine-deprived state (Fig. 1h). Cystine uptake is required for the synthesis of glutathione, a major antioxidant preventing elevated levels of reactive oxygen species (ROS). An oxidative, non-apoptotic, and iron-dependent form of regulated cell death caused by ROS-mediated massive lipid peroxidation (L-ROS) was recently described called ferroptosis ${ }^{21,22}$. Death of neuroblastoma cells with the oncogenic 'MYCN-high' background in a cystine-deprived state was averted by a specific inhibitor of ferroptosis or the lipophilic antioxidant, trolox (Fig. 1i, j). Supplementing cystine-free medium with the intracellular iron chelator, ciclopirox olamine (CPX), also prevented death of neuroblastoma cells with the 'MYCN-high' cellular background (Fig. 1k). Thus, intracellular cysteine depletion in a 'MYCNhigh' context induces neuroblastoma cell death by ferroptosis.

To investigate L-ROS formation in the context of cystine deprivation, we stained IMR5/75 cells with the lipid peroxidation sensor, C11-BODIPY, then flow cytometrically analyzed L-ROS in either 'MYCN-high' or 'MYCN-low' backgrounds with or without access to cystine from the growth medium. Cellular L-ROS was not altered by MYCN expression levels alone, but was approximately tenfold higher in the 'MYCN-high' than 'MYCN-low' background following cystine deprivation (Fig. 1I). Inhibiting ferroptosis with either ferrostatin-1 or liproxstatin-1 in the 'MYCN-high', cystine-deprived state rescued cells from massive L-ROS accumulation, as did treatment with the lipophilic antioxidant, trolox, or supplementing cystine-free medium with the intracellular iron chelator, CPX (Fig. 1m). Supplementing cystine-free medium with glutathione also averted L-ROS accumulation and cell death (Fig. $1 \mathrm{~m}$ ), pinpointing glutathione as a crucial factor preventing L-ROS accumulation and 
ferroptosis in the oncogenic 'MYCN-high' and cysteine-deprived context. Paradoxically, depletion of both glutamine and cystine delayed ferroptosis in the 'MYCN-high' state (Fig. 1n). It is widely thought that glutamine deprivation drives MYC-dependent cells into apoptosis by creating an intracellular glutamate deficiency ${ }^{16-19}$. Our data only show a slight selective effect of glutamine deprivation on cell death in the 'MYCN-high' state and no additive effect for depletion of glutamine and cystine. New data gathered in mouse embryonic fibroblasts show that glutaminolysis is essential for ferroptosis triggered by depletion of full amino acids or cystine alone ${ }^{23}$. Our findings together with new descriptions of the ferroptotic process establish a novel functional link between oncogenic $\mathrm{MYC}(\mathrm{N})$ and ferroptosis, and imply regulation by cysteine-dependent glutathione availability.

\section{Glutathione becomes an Achilles' heel}

Cells in the 'MYCN-high' state may be susceptible to redox imbalances and respond by maintaining high glutathione levels to prevent iron-dependent L-ROS formation and subsequent ferroptosis. MYC has been reported to upregulate glutathione in other cancer cells $^{24}$. Neuroblastoma cells in the 'MYCN-high' state had three- to fourfold higher intracellular glutathione levels (Fig. 2a) and twofold higher reduced:oxidized glutathione ratios (Fig. 2b), reducing intracellular ROS levels (Fig. 2c) compared with the 'MYCN-low' state. An unbiased high-throughput MYCN synthetic lethal siRNA screen (Fig. 2d) identified genes preferentially acting in the 'MYCN-high' state and protecting cells from ROS accumulation and ferroptosis. Top hits included six enzymes in glutathione metabolism, which are preferentially active in lipid peroxide detoxification (Fig. 2e-f and Extended Data Table 1). Massive reduction in cell number was selectively triggered in the 'MYCN-high' context (Fig. 2f, g) by individual knockdown of glutathione-disulfide reductase (GSR), glutathione peroxidase 4 or 6 (GPX4, GPX6), or glutathione S-transferase mu 1, mu 5, or kappa 1 (GSTM1, GSTM5, GSTK1). Knockdown of genes for the two enzymes catalyzing glutathione biosynthesis, glutamate-cysteine ligase catalytic subunit (GCLC) and glutathione synthetase (GSS) were synthetic lethal with $M Y C N$, but were not prioritized under the stringent criteria applied in our unsupervised statistical selection (Fig. 2h). Glutathione peroxidase (GPX4) was among our top hits, and is a potent negative regulator of ferroptosis $^{22}$. Co-treatment with the MYC-MAX inhibitor, 10058-F4, rescued cells in the 'MYCN-high' state from undergoing ferroptosis in response to RSL-3, a small molecule inhibiting GPX4 (Fig. 2i). Five neuroblastoma cell lines harboring various MYCN complements and expressing $\mathrm{MYC}(\mathrm{N})$ protein levels ranging from non-detectable to strong responded differently to cystine deprivation (Extended Data Fig. 2a, b). MYC(N) expression in these cell lines determined their response to RSL-3 treatment, with the SK-N-FI cell line, lacking detectable $\mathrm{MYC}(\mathrm{N})$ expression, being virtually resistant to ferroptosis induction (Fig. 
2j). Elevated cellular free iron levels have been suggested to predispose cancer cells to ferroptosis ${ }^{21,22}$, and oncogenic MYC induces iron uptake required for diverse enzymatic reactions in fast-proliferating cells by activating the transferrin receptor $(T F R C)^{25}$. We hypothesized that increasing the iron supply may boost ferroptotic cell death in MYCN-driven cells. Indeed, supplying iron enhanced the RSL-3 effect on the MYCN-amplified KELLY cell line by tenfold (Fig. 2k). Taken together, we identify multiple points in glutathione synthesis and metabolism, particularly detoxification of L-ROS, that are vulnerable in the 'MYCN-high' context, and show that ferroptosis is dependent on $\mathrm{MYC}(\mathrm{N})$ expression and enhanced by iron.

\section{Transsulfuration maintains glutathione}

Cellular cysteine can be oxidized from imported cystine or synthesized from methionine ${ }^{26}$. The solute carrier family 7 member 11 (SLC7A11) of the $x_{c}{ }^{-}$system imports cystine in exchange for glutamate ${ }^{27}$, whereas SLC7A5 imports methionine and branched chain amino acids in exchange of glutamine ${ }^{28}$ (Fig. 3a). Sulfasalazine and erastin are small molecule inhibitors of SLC7A11, from which erastin also inhibits SLC7A $5^{28-30}$. Selective induction of ferroptosis in 'MYCN-high' cells, compared to cells in the 'MYCN-low' state or with MYC(N) activity inhibited by 10058-F4, returned with erastin treatment (Fig. 3b and Extended Data Fig. 3a). Erastin also induced death in a similar pattern to RSL-3 in the five neuroblastoma cell lines expressing a panorama of oncogenic MYCN levels (Fig. 3b) that was boosted by iron supply (Extended Data Fig. 3b), demonstrating that erastin induces ferroptosis in the 'MYCN-high' cellular context. SLC7A5 knockdown revealed a MYCN synthetic lethal effect, whereas SLC7A11 knockdown had a similar effect in both 'MYCN-high' and 'MYCN-low' contexts (Fig. 3c), as did treatment with sulfasalazine (Extended Data Fig. 3c). We conclude that quantitatively reducing SLC7A11 antiporter activity does not limit cysteine availability. MYC(N)-selective induction of ferroptosis appears to be independent of cystine uptake by SLC7A11 and conferred by the import of methionine in exchange for glutamine by SLC7A5.

Cysteine is synthesized from methionine via transsulfuration, whereby homocysteine, a methionine cycle intermediate, and serine are combined to cystathionine and then converted to cysteine ${ }^{26}$ (Fig. 3a). Four genes encoding enzymes key for converting methionine to cysteine were synthetic lethal with MYCN (Fig. 3d), as were two methyltransferases that may indirectly increase homocysteine levels (Extended Data Fig. 3c). We interpret these findings to mean that transsulfuration provides a critical additional cysteine source for $M Y C N$-amplified cells. Supporting this interpretation, supplementing media with either the homocysteine or cystathionine intermediate prevented cystine deprivation-induced ferroptosis in IMR5/75 cells in the 'MYCN-high' state (Fig. 3e) and cell lines maintaining oncogenic MYCN activity (Fig. 3e, Extended Data Fig. 4). Activating 
transsulfuration appears to require cell type-specific factors present in cells that have naturally evolved dependency on oncogenic MYCN signaling, since supplying homocysteine or cystathionine to a cell model expressing the MYCN transgene in the normal diploid MYCN background did not avert cystine deprivation-induced ferroptosis (Fig. 3e). Pharmacologically inhibiting intracellular cysteine synthesis with an inhibitor of cystathionine-gamma-lyase (PPG) sensitized cells with naturally evolved MYCN dependency and active transsulfuration, but not ectopically expressed MYCN and inactive transsulfuration, to erastin- or sulfasalazine-induced cell death (Fig. 3f). Downregulating S-adenosylhomocysteine hydrolase $(A H C Y)$ reduced glutathione levels and ratios of reduced:oxidized glutathione states (Fig. 3g, h). Colony formation by cells in the 'MYCN-high' state was impaired relative to the amount of $A H C Y$ downregulation (Fig. $3 \mathrm{~g}$ ). In contrast, $A H C Y$ downregulation had no effect on glutathione levels or colony formation (Fig. $3 \mathrm{~g}$ ) in cells with a diploid MYCN background and inactive transsulfuration. Similar to erastin and RSL-3, pharmacologically suppressing homocysteine synthesis by inhibiting AHCY with $\mathrm{D} 9^{31}$ in five neuroblastoma cell lines induced cell death in a manner positively correlated with MYCN expression level (Fig. 3i). Thus, neuroblastoma cells with oncogenic $M Y C N$ activity use the methionine cycle and transsulfuration as an internal cysteine source for glutathione biosynthesis to evade ferroptosis.

\section{Oncogenic MYCN induces transsulfuration}

Our data show that both cystine import and intracellular cysteine synthesis achieve the intracellular state supportive of oncogenic $\mathrm{MYC}(\mathrm{N})$-driven growth without endangering the cell to ferroptosis. To understand how cysteine metabolism and redox homeostasis are adapted by oncogenic $\mathrm{MYC}(\mathrm{N})$ activity, we analyzed differential gene expression in synchronized cells in 'MYCN-high' and 'MYCN-low' states ${ }^{32}$. Synchronizing the cell cycle separated indirect effects changing with cell cycle phases and related to accelerated proliferation from oncogenic $M Y C(N)$ actions pervasive throughout the cell cycle (Fig. 4a, Extended Data Fig. 5a). Expression of cystathionine-beta-synthase (CBS), AHCY and phosphoglycerate dehydrogenase $(P H G D H)$, three key enzymes for transsulfuration, was elevated in the 'MYCN-high' state across all cell cycle phases, while expression of the cysteine uptake-controlling $\mathrm{x}_{c}^{-}$system (SLC7A11) was unaffected by MYCN expression level (Fig. 4a and Extended Data Fig. 5a). Control by oncogenic MYC(N) of the three transsulfuration enzymes was confirmed in a panel of 29 neuroblastoma cell lines with diverse $M Y C(N)$ expression (Extended Data Fig. 5b). Histone marks associated with active transcription depict MYCN as an amplifier of active $A H C Y$ transcription in cells with oncogenic $\mathrm{MYC}(\mathrm{N})$ activity (Extended Data Fig. 6a, b). CBS regulation appears from ChIPseq analysis to be orchestrated by both activating and silencing histone modifications. The 
CBS regulatory region was bound by MYCN and harbored activating histone marks, while almost completely lacking silencing marks, in cells with oncogenic MYCN (Fig. 4b, c, Extended Data Fig. 7a, b). The CBS promoter was predominantly occupied by repressive marks in cells harboring diploid $M Y C N$ and lacking detectable $M Y C(N)$ expression (Fig. 4b, $\mathrm{c}$, Extended Data Fig. 7a, b). Ectopic MYCN expression in the MYCN diploid background induced expression of $A H C Y$ and other MYCN targets, while $C B S$ remained epigenetically repressed (Extended Data Fig. 7c). Differences in CBS expression in primary neuroblastomas correlated with histone modifications and methylation of intronic CpGs dependent on the genomic MYCN status (Fig. 4d, Extended Data Fig. 8). These data paint a picture where $A H C Y$ is constitutively active in neuroblastoma cells, but further enhanced by MYCN, while CBS is more strictly epigenetically controlled and may require prior release from epigenetic repression before MYCN-dependent activation can occur.

Global gene expression profiles from 498 primary neuroblastomas ${ }^{33}$ also showed significantly higher $C B S$ and $A H C Y$ expression in $M Y C N$-amplified neuroblastomas (Fig. 4e, g). MYCN expression strongly correlated with $C B S$ and $A H C Y$ expression in tumors, and high $C B S$ or $A H C Y$ levels were associated with poorer overall patient (Fig. 4f, $g$ and Extended Data Figure 9). Mass spectrometry-based global proteomes from six primary neuroblastomas also showed that $\mathrm{CBS}, \mathrm{AHCY}$ and $\mathrm{PHGDH}$ expression was higher in tumors harboring MYCN amplifications (Fig. 4h). CBS and $A H C Y$ expression correlated with $P H G D H$ expression in tumors as well as with TP53 expression, which is only mutated in $2 \%$ of primary neuroblastomas $^{34}$ (Fig. $4 \mathrm{~g}$ ). TP53 is a transcriptional target of $\mathrm{MYCN}^{10,35}$ and is known to suppress cystine uptake via the $\mathrm{x}_{\mathrm{c}}^{-}$system by SLC7A11 repression ${ }^{36}$. SLC7A11 expression did not correlate with either MYCN amplification, TP53 expression or other risk factors for poor patient outcome (Fig. 4g). Combining these data with the unchanged expression of the $\mathrm{x}_{\mathrm{c}}^{-}$system in cells in either the 'MYCN-high' or 'MYCN-low' states, confirms a lack of direct control of cystine uptake by oncogenic MYCN activity, but may indicate that processes simultaneously up- and downregulating SLC7A11 may be holding expression steady in a varying MYC $(N)$ context. Although the mechanisms upregulating the enzymes for transsulfuration in a cell with oncogenically active $\mathrm{MYC}(\mathrm{N})$ may require indirect components, $\mathrm{MYC}(\mathrm{N})$ drives increased transsulfuration activity, rather than cysteine import, in tumor cells to maintain the cellular cysteine supply for glutathione synthesis.

The 'MYCN-high' state also supported enhanced methionine, glutamine and iron uptake throughout the cell cycle (Extended Data Fig. 5a). Expression of transsulfuration enzymes in primary neuroblastomas also correlated with transporters for glutamine, methionine, and iron uptake (Fig. 4g). Enhanced expression of transporter proteins for methionine, glutamine, and iron was confirmed in $M Y C N$-amplified tumor proteomes (Fig. 5h). Because genes mediating cytosolic glutaminolysis ${ }^{37}$ were barely expressed in neuroblastoma 
cells and tumors (Extended Data Fig. 5c, Fig. 4g), it is unlikely that imported glutamine is used as a source for glutamate in cysteine exchange via the $\mathrm{x}_{\mathrm{c}}{ }^{-}$system and glutathione synthesis occurring in the cytosol ${ }^{24}$. Higher expression of the mitochondrial glutaminolysis isoform ${ }^{38}$ in $\mathrm{MYCN}$-amplified tumor transcriptomes and proteomes (Fig. $4 \mathrm{~g}, \mathrm{~h}$ ) suggests that glutamate synthesis is required only in the neuroblastoma cell mitochondria. Expression of two enzymes regulating the glutathione biosynthesis (GCLC) and redox cycle (GSR) positively correlated with MYC $(\mathrm{N})$ expression in neuroblastoma cell lines (Extended Data Fig. $5 b)$ and were more strongly expressed in the $M Y C N$-amplified tumor transcriptomes and proteomes (Fig. 4g, h). Oncogenic MYC(N) activity appears to fuel hyperactive glutathione redox activity by increasing import of methionine, upregulating three key enzymes for transsulfuration to increase intracellular cysteine and activating glutathione biosynthesis and metabolism.

Elevated MYCN expression from a normal MYCN locus occurs in low-risk neuroblastomas, particularly in metastatic disease that tends to regress spontaneously in children under 18 months of age ${ }^{10}$. Glutathione biosynthesis and redox activity, which climbs in the $M Y C N$-amplified scenario, remained unchanged with fluctuating MYCN in low-risk, metastatic neuroblastomas (Fig. 4i). Moreover, intronic CpG methylation epigenetically silenced $C B S$ in low-risk, metastatic cases with low CBS expression (Extended Data Fig. 8), indicating that transsulfuration is not available to increase internal cysteine for glutathione biosynthesis.

\section{Discussion}

We show that MYCN sensitizes neuroblastoma cells to an oxidative, non-apoptotic, iron-dependent regulated type of cell death when intracellular cysteine availability for glutathione synthesis is limited. We propose that a 'MYCN-high' state in neuroblastoma cells sensitizes them to lipid peroxidation, which in combination with an 'acute' intracellular cysteine reduction, triggers metabolic sabotage ${ }^{39}$ and massive ferroptotic cell death that may explain spontaneous neuroblastoma regression (Fig. 5). Robust molecular markers for tissues undergoing ferroptosis are not yet known ${ }^{40}$. However, our study shows transsulfuration, glutathione redox activity and methionine import to be crucial to escape ferroptosis, and correlated expression of key genes for these processes with MYCN expression in tumors. Expression for these key genes did not correlate with MYCN expression in low-risk primary neuroblastomas that frequently undergo spontaneous regression, and repressive histone marks at $C B S$ regulatory regions and hypermethylated intronic CpGs were associated with low CBS expression in the low-risk neuroblastomas. The absence of MYCN-dependent epigenetic regulation of $C B S$ in a low-risk neuroblastoma compounded by reduced import of methionine would prevent the tumor from developing the 
hyperactive glutathione biosynthesis and redox activity it needs to guard against ferroptosis. Spontaneous regression may be the physiological resolution of this cellular state. Unlike lowrisk tumors, $\mathrm{MYCN}$-amplified neuroblastomas appear to metabolically adapt to survive events resulting in intracellular cysteine reduction, such as TP53 activation and SLC7A11 repression ${ }^{36}$ following telomere crisis. The cysteine requirement of cancers dependent on oncogenic $\mathrm{MYC}(\mathrm{N})$ activity creates a previously unknown Achilles' heal that could be exploited to selectively induce ferroptosis for treatment. Our findings identify cysteine uptake, intracellular methionine conversion to cysteine, the L-ROS-specific scavenging system, and the cellular free iron pool as vulnerabilities in cancer cells driven by oncogenic $M Y C(N)$ activity such as $M Y C N$-amplified neuroblastomas. 

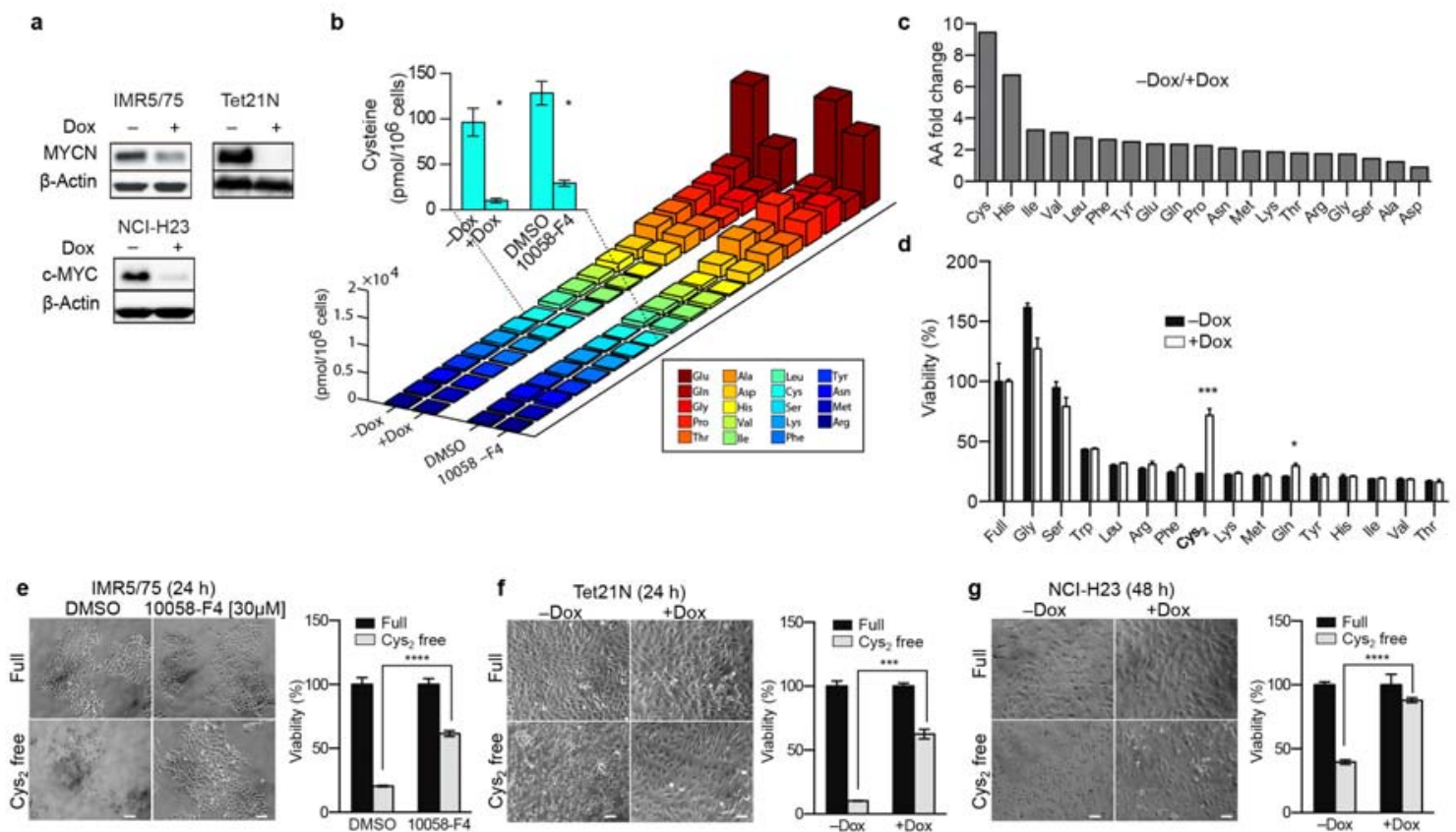

h
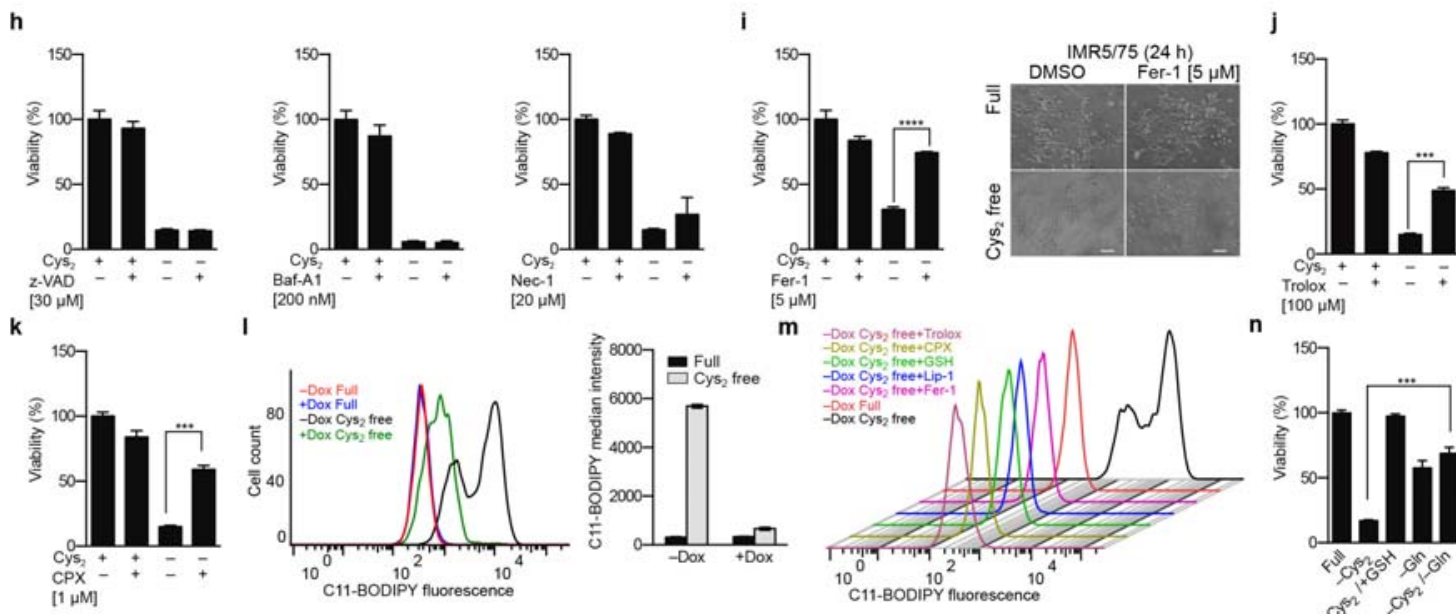

Figure 1. Cystine addiction of MYC(N)-expressing neuroblastoma and lung cancer cells

a, Representative western blot of neuroblastoma (IMR5/75, Tet21N) and lung cancer ( $\mathrm{NCl}-\mathrm{H} 23$ ) cells upon MYC(N) knockdown using doxycycline (- Dox, high expression; +Dox, low expression).

b, c, Intracellular amino acid quantification (b) and ranked fold changes (c) in IMR5/75 reveal cysteine (Cys) reduction after MYCN inhibition (+Dox or 10058-F4, $96 \mathrm{~h}$ ).

d, Standardized viability of IMR5/75 after single amino acid depletions (48 h) reflects cell death resistance of cystine (Cys ${ }_{2}$ )-deprived 'MYCN-low' cells.

e-g, $\mathrm{Cys}_{2}$ addiction is linked to 'MYC(N)-high' state; Scale: $50 \mu \mathrm{m}$, mean of triplicates standardized to NT (full medium).

h-k, Cys $_{2}$ deprivation-induced death of IMR5/75 is preventable using ferroptosis inhibitors.

I-n, Flow cytometry confirms L-ROS accumulation in Cys $_{2}$-deprived 'MYCN-high' IMR5/75 (I), reduction by ferroptosis inhibitor treatments or GSH supplementation $(\mathbf{m})$ and maintained viability $(\mathbf{n})$ when supplied with GSH or glutamine (Gln, $24 \mathrm{~h}$ ). Data are representative of at least two (e-g), three $(\mathbf{d}, \mathbf{h}-\mathbf{n})$ or more (b) biological replicates (means $\pm \mathrm{SD}$ ). two-tailed Student's t-test: ${ }^{*} \mathrm{P} \beth<\sqsupset 0.05$, ${ }^{* *} \mathrm{P} \sqsupset<\sqsupset 0.01,{ }^{* * *} \mathrm{P} \sqsupset<\sqsupset 0.001,{ }^{* * *} \mathrm{P} \beth<\sqsupset 0.0001$. 


\section{a}

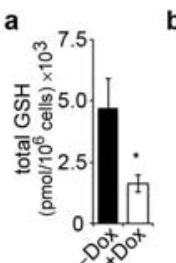

C
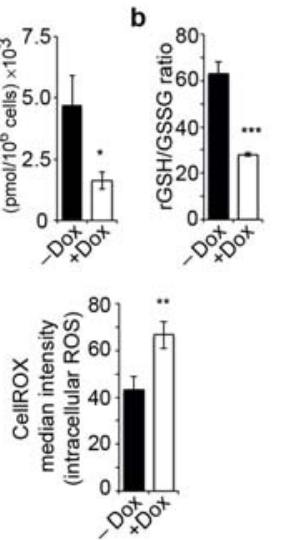

d

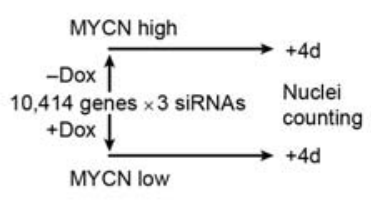

e

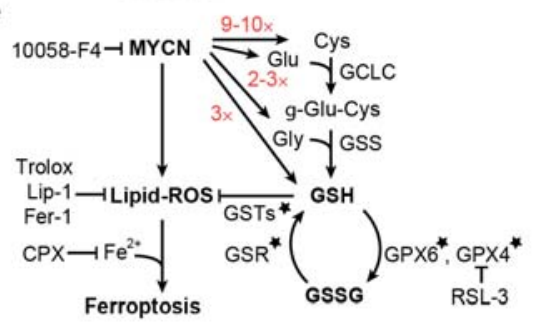

h

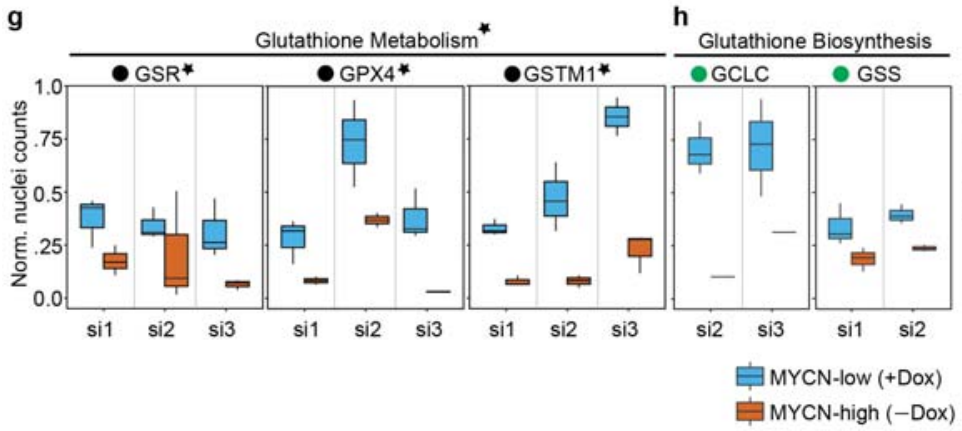

f

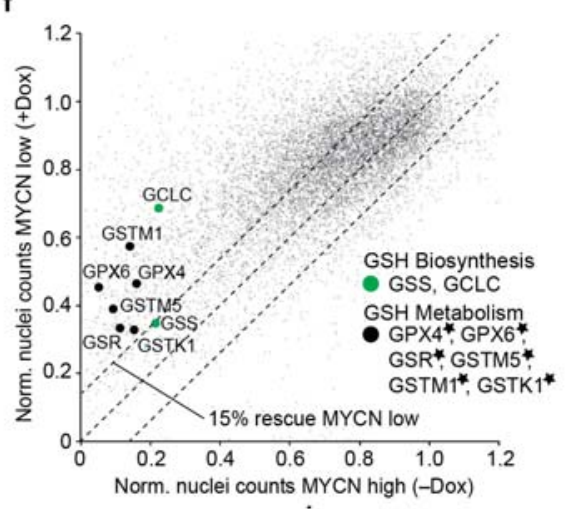

j 120
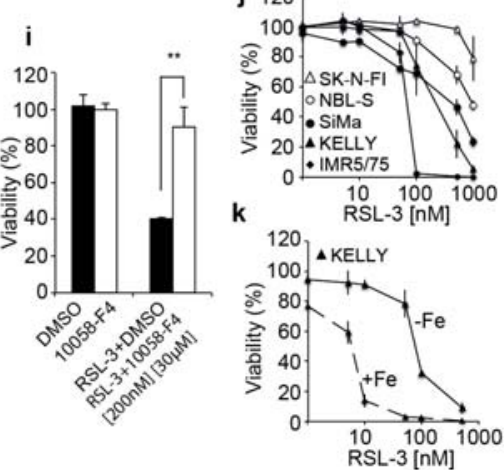

Figure 2. Inhibition of GSH metabolism is synthetic lethal with high MYCN

$\mathbf{a}, \mathrm{HPLC}$ reveals elevated intracellular glutathione, and $\mathbf{b}$, ratio of reduced:oxidized GSH expressed as GSH equivalents in 'MYCN-high' IMR5/75. c, Flow cytometry confirms intracellular ROS reduction (lower CellROX intensity) in 'MYCN-high' state; three biological replicates; means \pm SD.

d-h, MYCN synthetic lethal druggable genome-wide siRNA screening approach in IMR5/75 (d); MYCN effects on L-ROS formation and intracellular amino acid levels (fold changes in red), the two-step biosynthesis of GSH and GSH metabolism; *top MYCN synthetic lethal hits of GSH metabolism, false discovery rate (FDR) of 0.2; action of ferroptosis inhibitors (CPX, Fer-1, Lip, Trolox and 10058-F4) and class II ferroptosis inducer (RSL-3) as indicated (e); $\mathbf{f}$, Effects of individual siRNAs (grey dots): 'MYCNhigh' vs. 'MYCN-low', including key players (median of 2-3 siRNAs) of $\mathbf{g}$, top MYCN synthetic lethal hits $\left(^{\star}\right)$ of GSH metabolism (black) and $\mathbf{h}$, GSH biosynthesis (green).

i-k, 10058-F4 treatment prevents RSL-3-induced cell death in IMR5/75 (72 h) (i); MYCN expression level of neuroblastoma cell lines reflects capacity to resist RSL-3-induced ferroptosis: cells with MYCN-amplification (black symbols), moderate MYCN expression, (white circles) and lack thereof (white triangles) (j), Fe supplementation $(25 \mu \mathrm{g} / \mathrm{ml})$ enhances RSL-3- induced death in KELLY $(\mathbf{k}) . \mathrm{n}=$

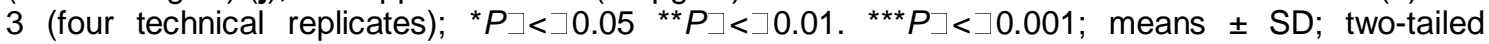
Student's t-test. 


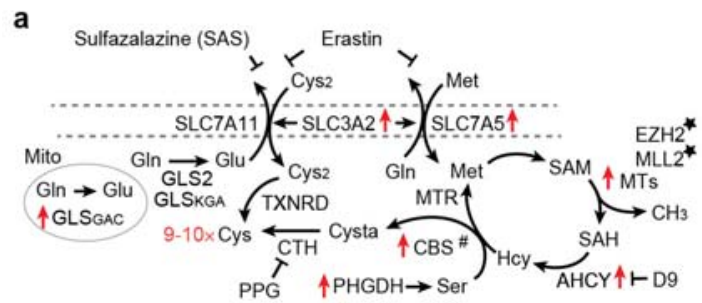

d

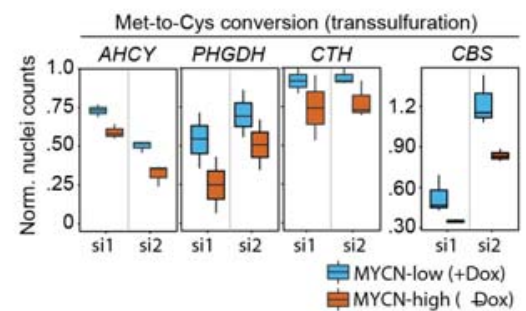

f
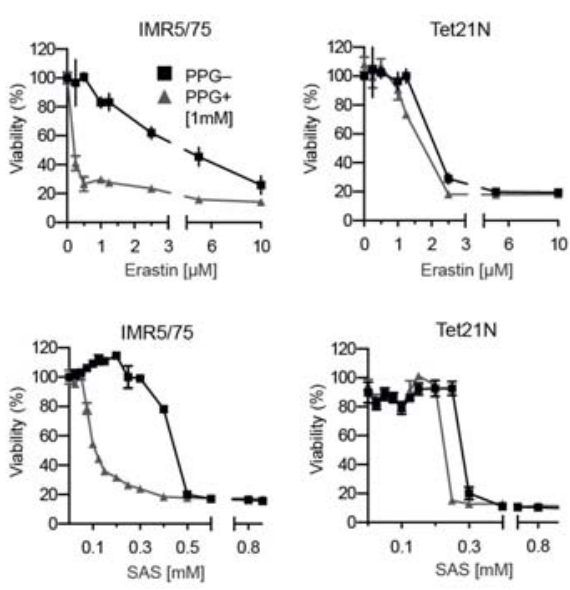
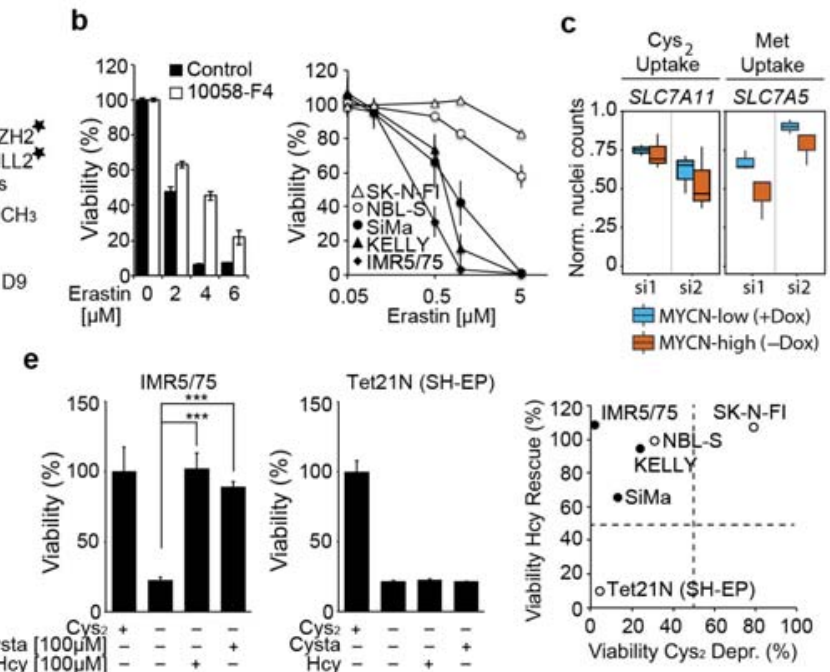

g
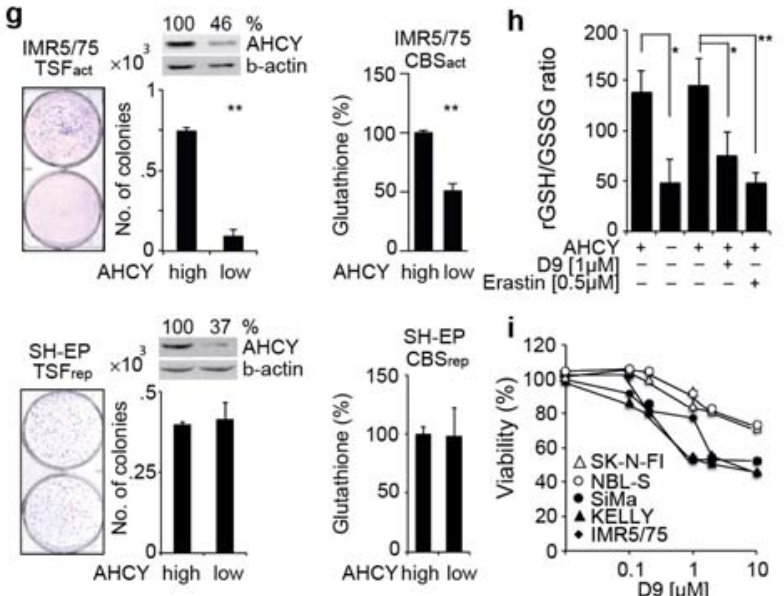

Figure 3. MYCN activates transsulfuration genes controlling Met-to-Cys conversion in MYCNamplified cells

a, Illustration of Cys $s_{2}$ and Met uptake/metabolism, and transsulfuration $\left({ }^{\#}\right)$, Mito = mitochondria, MTs = methyltransferases, Cysta = cystathioinine; ${ }^{\star}$ : top hits from MYCN siRNA screen; red arrows: genes transcriptionally activated by MYCN in IMR5/75 (FDR 0.001, using likelihood ratio testing) with Cys fold changes (red).

b, Erastin treatment (72 h) enables cell death in 'MYCN-high' IMR5/75, which is inhibited by 100058$\mathrm{F} 4$, and proves more potent in neuroblastoma cell lines with high MYCN activity; $n=3$ (four technical replicates).

c, d, IMR5/75 MYCN siRNA screen shows MYCN-independent SLC7A11 knockdown effect but MYCN synthetic lethality with knockdown of $S L C 7 A 5$ (c) and of key transsulfuration genes (d); two individual siRNAs.

e, Homocysteine (Hcy) and Cysta supply prevent $\mathrm{Cys}_{2}$ deprivation-induced ferroptosis in 'MYCN-high' IMR5/75 and cell lines with naturally evolved MYCN dependency; $n=3$ (four technical replicates).

f, Inhibition of Cys synthesis (PPG) enables erastin- or SAS- induced cell death in MYCN- dependent IMR5/75, unlike in ectopically MYCN expressing TET21 N, $n=3$, representative experiment is shown.

$\mathbf{g}-\mathbf{i}$, AHCY inhibition (knockdown or D9) reduces colony formation, glutathione synthesis $(\mathbf{g})$ and, like erastin, the $\mathrm{rGSH} / \mathrm{GSSG}$ ratio in IMR5/75 (h). D9 is more potent in cell lines with high $\mathrm{MYC}(\mathrm{N})$ expression (i); $\mathrm{n}=3$; ${ }^{*} P \_<\_0.05{ }^{* *} P \_<\_0.01{ }^{* * *} P<0.001$; means $\pm \mathrm{SD}$; two-tailed Student's t-test. 
a
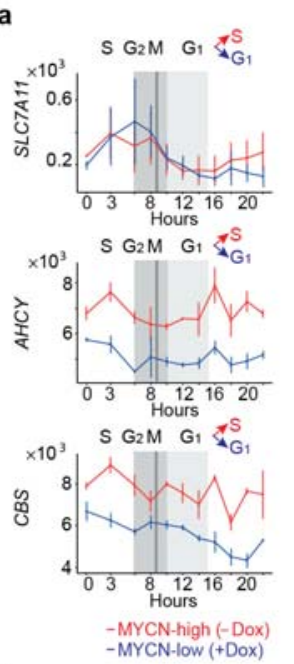

e
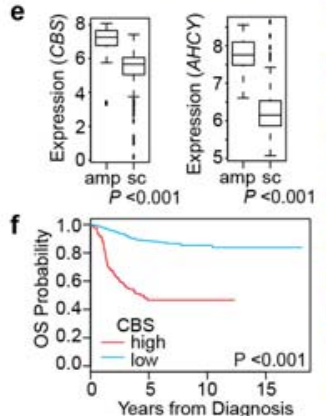

b

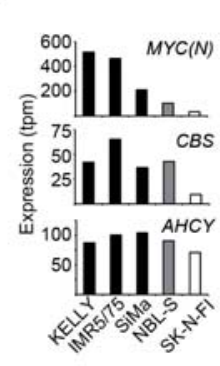

g
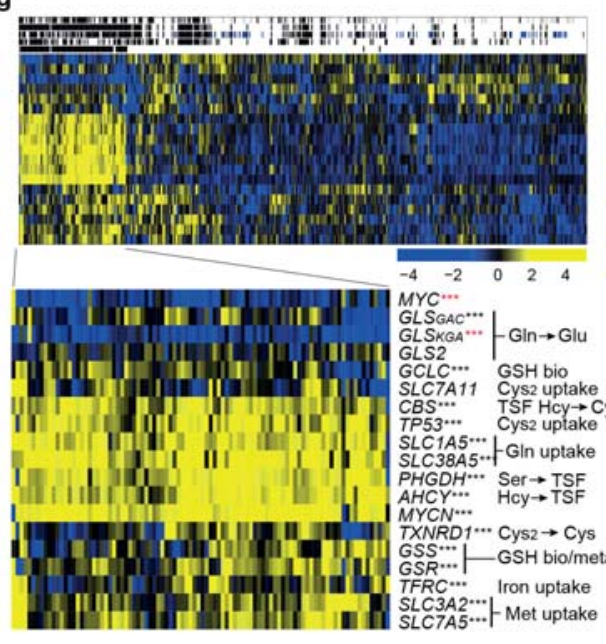

c
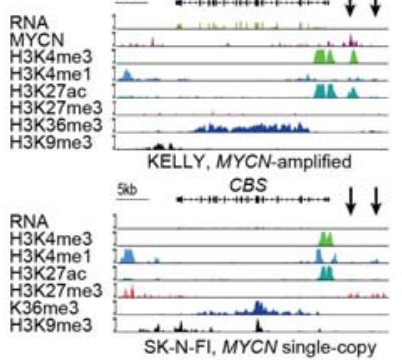

KELLLY, MYCN-amplified

MYC

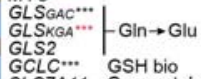

GCLC... GSH bio

SLC7A11 Cys2 uptake
CBS…

TP53... Cys uptake

SLC1A5 … - Cys2 uptake

PHGDH
AHCY...

MYCN …

TXNRD $1 \cdots$ Cys $2 \rightarrow$ Cys
GSS $\cdots$ GS
GSR $\cdots$ GSH bio/meta

TFRC....... Iron uptake

SLC $. .12 \ldots \mid$ Iron uptake
SLC7A5 … Met uptake d

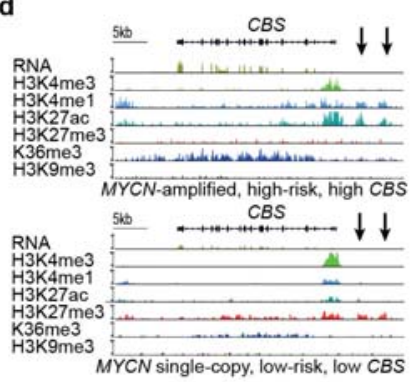

h

h

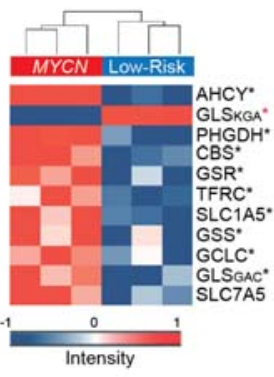

MYCN amp ( $n=92) \quad$ MYCN sc stage $4 s(n=48)$

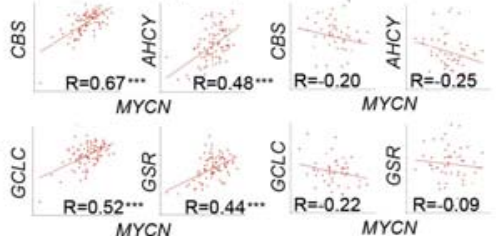

Figure 4. Differential anti-ferroptotic activities in regressing and progressing neuroblastomas

a, Expression of transsulfuration genes is linked to MYCN expression level during cell cycle progression, unlike SLC7A11 expression (IMR5/75, RNA-seq); means \pm SD; differentially expressed genes were identified using likelihood ratio testing.

b, mRNA expression of cells reflecting diverse $M Y C(N)$ expression levels.

c, d, mRNA expression (RNA-seq) and input normalized read counts of histone modifications and MYCN (ChiP-seq) at the CBS locus in representative MYCN-amplified and MYCN single-copy cells (c) and tumors (d).

e, MYCN status-dependent expression in 498 primary neuroblastomas (92 MYCN-amplified, 406 MYCN single-copy tumors, RNA-seq), Wilcoxon rank-sum test.

f. Kaplan-Meier overall survival estimates for subgroups defined by $C B S$ expression, high $C B S$ $(\mathrm{n}=123)$; low CBS $(\mathrm{n}=375)$.

g, Gene expression heatmap of subgroups hierarchically clustered with average linkage and noncentralized correlation distance function; row: transcript, column: sample, HR = high-risk, IR/LR = intermediate/low-risk according the German NB2004 study, amp = amplified, sc = single-copy, *** (black) $P<0.001$ higher and ${ }^{* * *}$ (red) $P<0.001$ lower gene expression, Wilcoxon rank-sum test.

h, Mass spectrometry reveals differential expression of proteins mediating GSH synthesis/metabolism and GSH-relevant amino acid uptake/metabolism: $M Y C N$-amplified vs. low-risk $M Y C N$ single-copy tumors, * (black) $P<0.05$ higher protein, * (red) $P<0.05$ lower protein expression using t-test, intensities as z-scores.

i, Pearson's correlation analysis of $M Y C N$ expression and GSH synthesis/metabolism and transsulfuration genes in MYCN-amplified ( $n=92)$ and $M Y C N$ single-copy stage $4 s(n=48)$ tumors. 


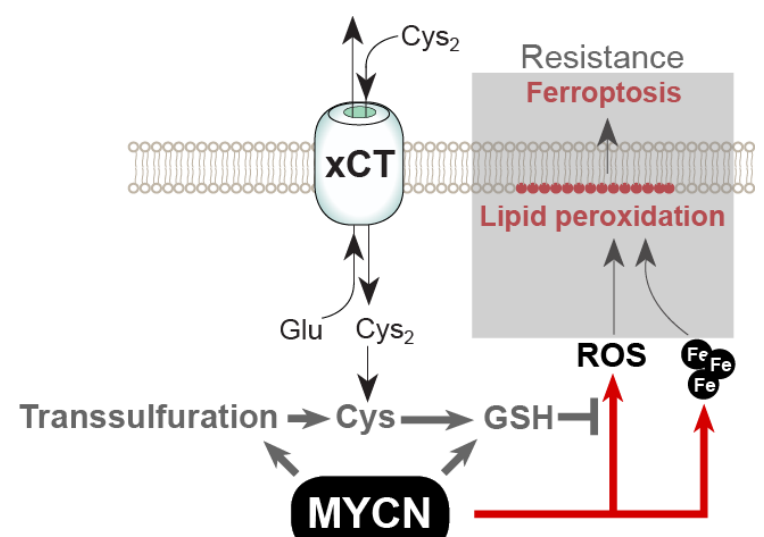

Figure 5. MYC(N)-mediated ferroptosis resistance

Cells with oncogenic $M Y C(N)$ respond to reduction of cysteine levels with massive lipid peroxidation resulting in ferroptosis, an oxidative, non-apoptotic and iron-dependent type of cell death. Deregulation of key components acting in transsulfuration, glutathione redox activity (grey arrows) and methionine import provides MYCN-driven neuroblastoma cells and tumors essential escape mechanisms to ferroptosis. 


\section{Extended data figures}

a

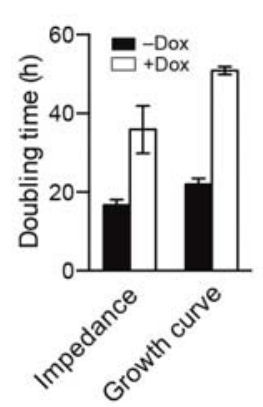

b

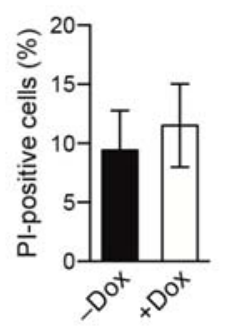

c

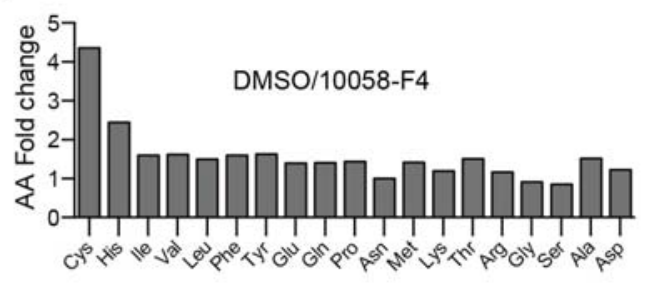

\section{Extended data figure 1}

a, Doubling time calculation from exponential growth curve quantified by FACS and impedance using the RCTA system for 'MYCN-high' (-Dox) and 'MYCN-low' (+Dox) IMR5/75 cells. Cell proliferation curves of exponentially growing cells (two biological replicates; means $\pm \mathrm{SD}$ ).

b, Viability quantification using FACS and propidium iodide staining in exponentially growing cells: Dox vs. +Dox.

c, Fold changes of intracellular amino acid levels $24 \mathrm{~h}$ after 10058-F4 treatment (normalized with DMSO control). 

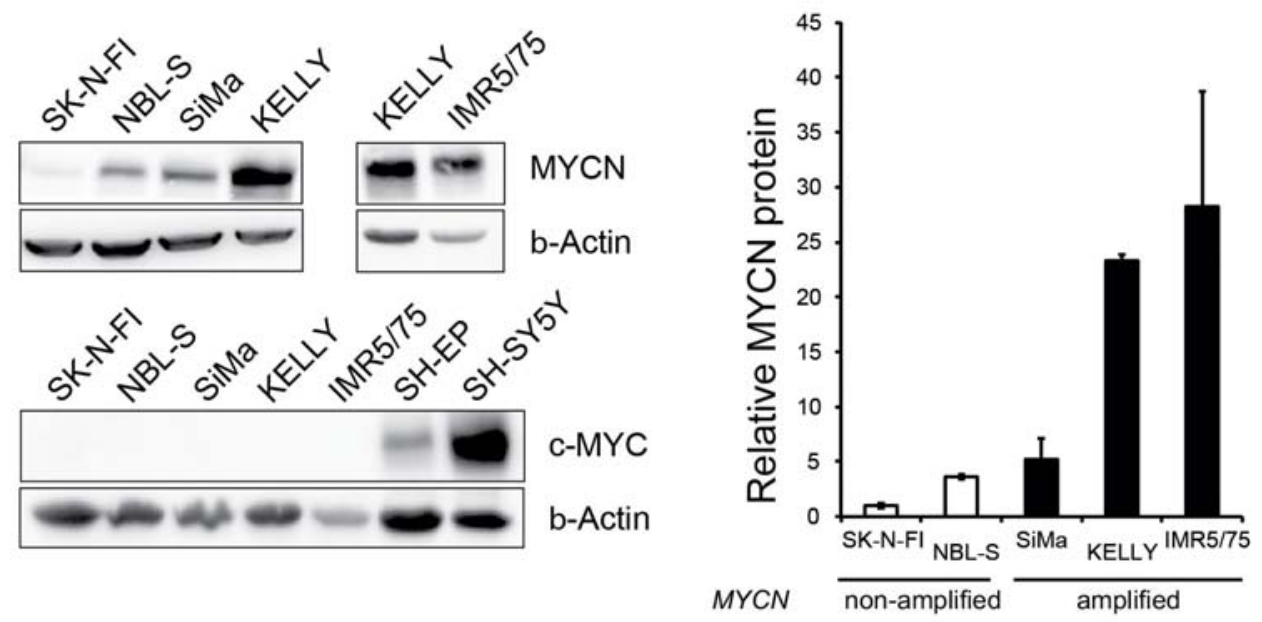

b

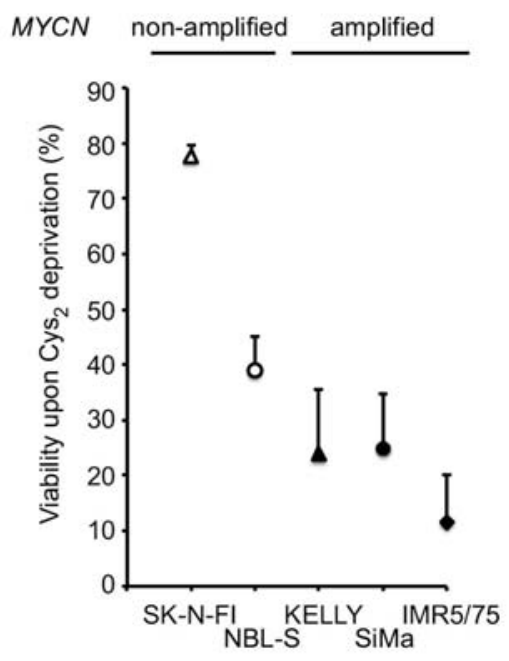

\section{Extended data figure 2}

a, MYCN and C-MYC protein expression in five representative neuroblastoma cell lines with diverse MYC(N) expression levels (western blot). $\sqsupset$-actin was used as loading control. SH-SY5Y and SH-EP cells were used as positive control for high and low c-MYC-expressing neuroblastoma cell lines, respectively. Quantification of MYCN protein levels; the lowest MYCN/ $\neg$-actin ratio in SK-N-FI was set to one and the higher MYCN/ - -actin ratios of the other cell lines calculated as fold changes. Means and standard deviations based on three independent western blot experiments.

b, Relative viability changes (\%) after 48 h Cys 2 deprivation (\% CTB fluorescence reads at 540/580 $\mathrm{nm}$ ) for five neuroblastoma cell lines with diverse MYC(N) expression levels. Mean and standard deviation of three independent experiments are depicted. $M Y C N$-amplified cell lines (black symbols), MYCN single-copy NBL-S cells with moderate MYCN expression (white circles), and SK-N-FI cells lacking $\mathrm{MYC}(\mathrm{N})$ expression (white triangles); $\mathrm{n}=3$ (4 technical replicates). 
a

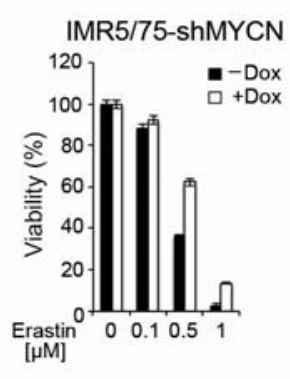

b

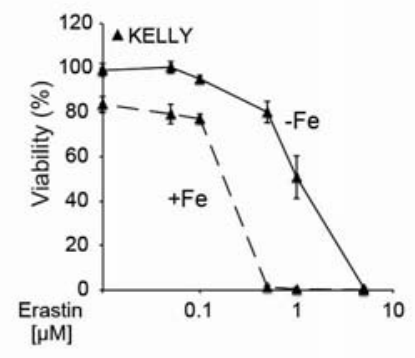

c

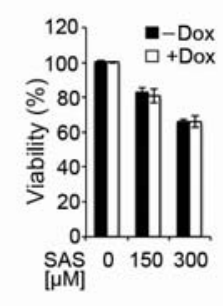

d

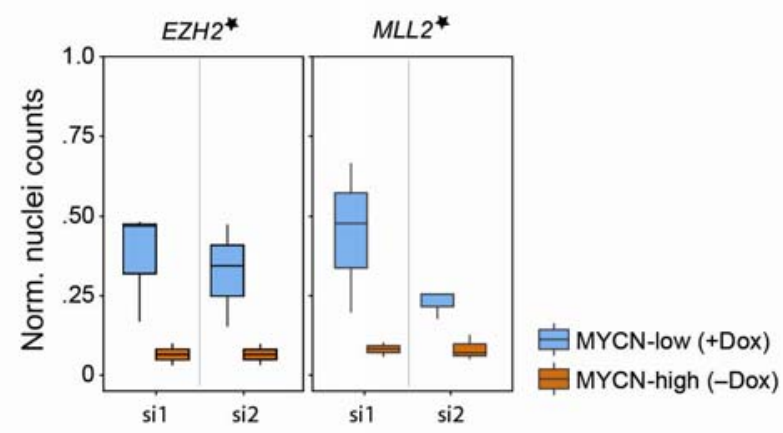

Extended data figure 3

a, MYCN synthetic lethal effect of class I ferroptosis inducer (FIN) erastin on viability of MYCNamplified IMR5/75 cells with Dox-inducible expression of a MYCN shRNA; $n=3$ (4 technical replicates; means $\pm \mathrm{SD}$ ).

b, Viability of KELLY cells upon treatment with erastin w/o iron $(25 \mu \mathrm{g} / \mathrm{ml}$, Venofer); $\mathrm{n}=3$ (4 technical replicates; means $\pm \mathrm{SD}$ ).

c, Viability of IMR5/75 cells upon treatment with sulfusalazine (SAS); $n=3$ (4 technical replicates; means \pm SD).

d, Two methyltransferases that may indirectly increase homocysteine levels are synthetic lethal with high MYCN (from the MYCN siRNA screen; ${ }^{\star}$ FDR of 0.2 ). 

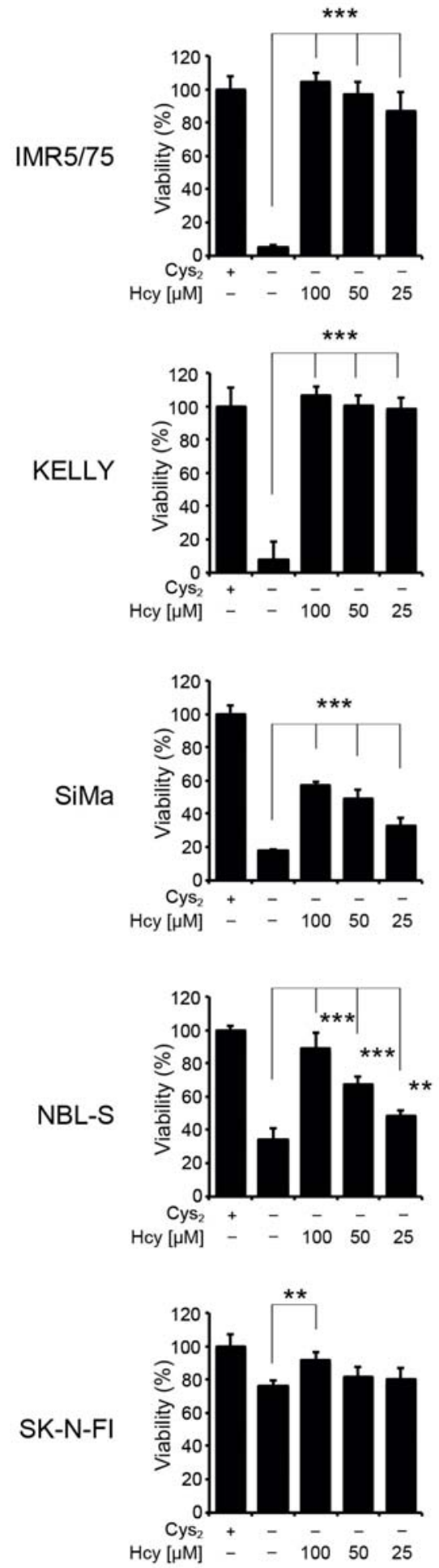
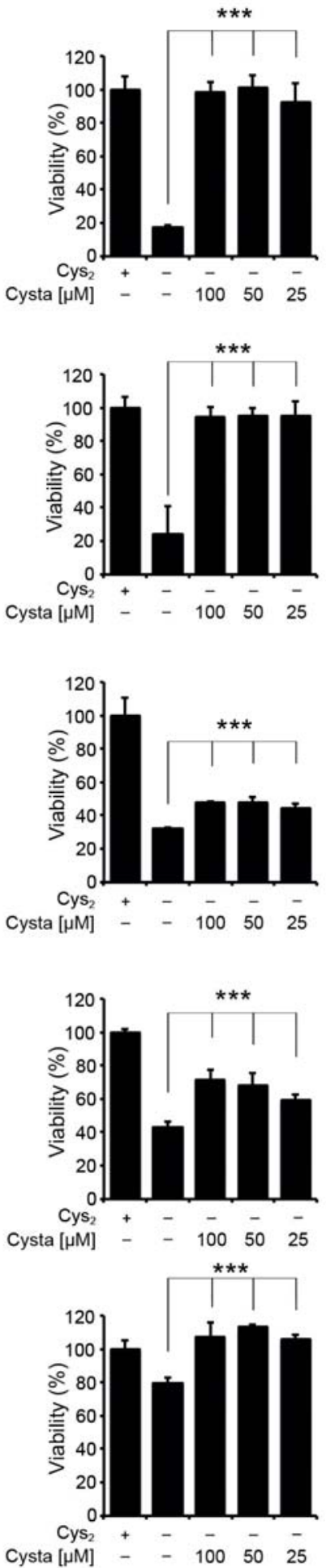

Extended data figure 4

Viability response of $\mathrm{Cys}_{2}$-deprived neuroblastoma cell lines with different levels of $\mathrm{MYC}(\mathrm{N})$

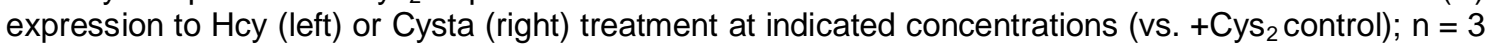
(4 technical replicates; means $\pm \mathrm{SD}$ ); ${ }^{* *} P \sqsupset<\sqsupset 0.01,{ }^{* * *} P \sqsupset<\sqsupset 0.001$; two-tailed Student's t-test. 
a

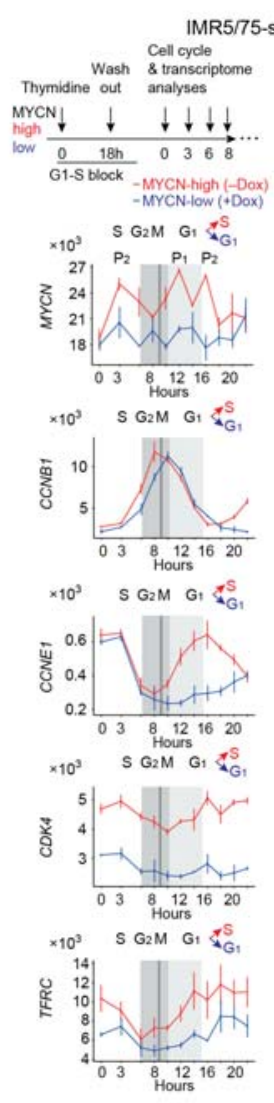

b

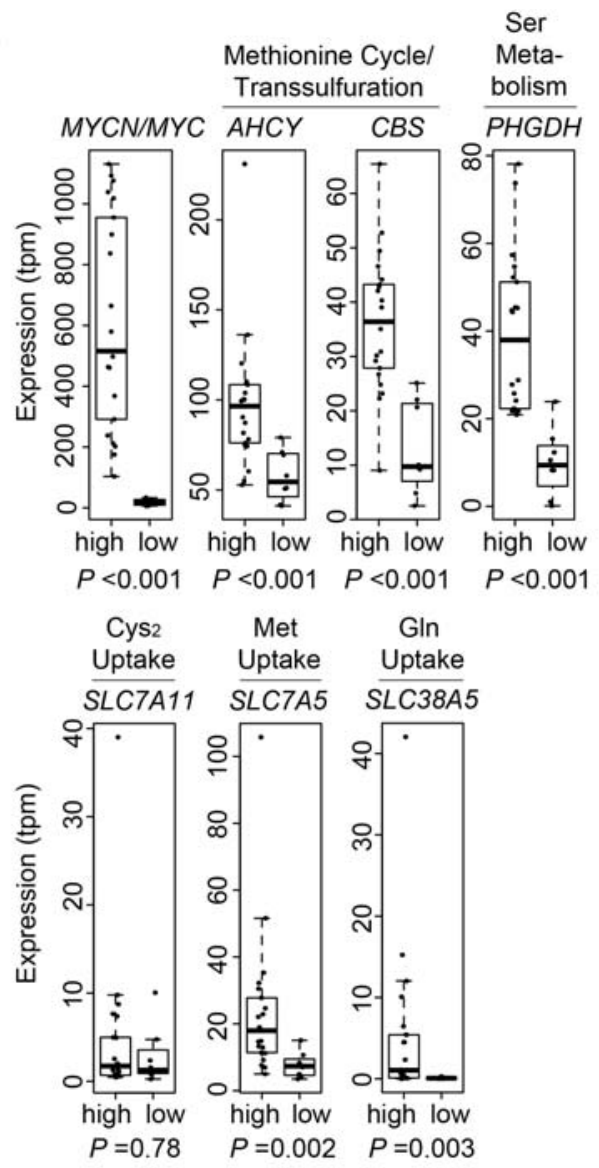

Glutathione Biosynthesis/

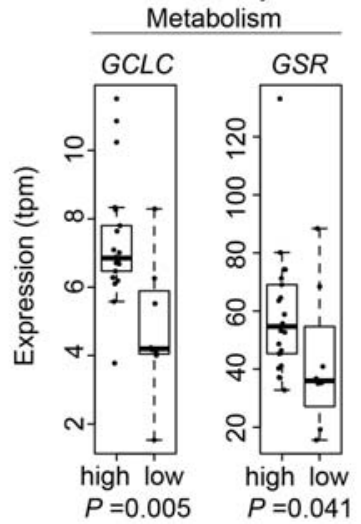

\section{Extended data figure 5}
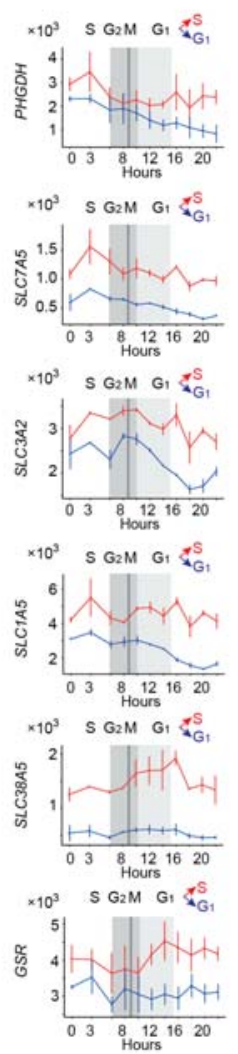

c

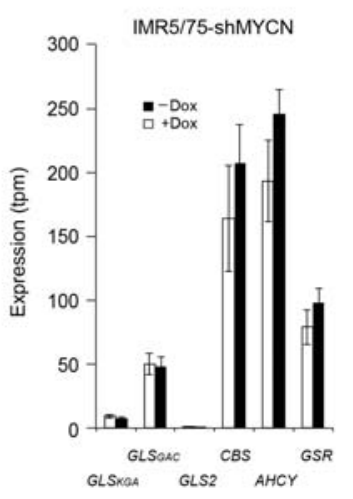

a, Time-resolved gene expression profiles during cell cycle progression: MYCN-high vs. MYCN-low IMR5/75 cells. To distinguish pervasive MYCN functions from indirect effects related to accelerated cell proliferation, 'MYCN-high' and 'MYCN-low' cells were synchronized using a thymidine block for 18 $\mathrm{h}$. Thymidine block-released cells were harvested every $2 \mathrm{~h}$ over a period of $22 \mathrm{~h}$ for transcript profiling using RNA-seq and cell cycle analysis (flow cytometry). The MYCN gene expression profile reveals two cell cycle-related peaks (P1 before G1-S transition and P2 before S-G2/M). Characteristic profiles for a pervasively MYCN-induced gene (CDK4) and two MYCN-induced cell cycle-regulated genes (CCNE1 for G1-S transition and CCNB1 for G2-M transition); means \pm SD.

b, Expression of GSH biosynthesis/metabolism, transsulfuration, and amino acid uptake/metabolism genes in 29 neuroblastoma cell lines as defined by RNA-seq: MYC(N)-expressors vs. MYC(N)-nonexpressors (c-MYC- and MYCN-expressing cell lines combined), Wilcoxon rank-sum test.

c, Expression of glutaminolysis genes (mitochondrial $G L S_{G A C}$; cytosolic $G L S_{K G A}$ and GLS2) as compared to MYCN-regulated genes (CBS, AHCY, GSR) in 'MYCN-high' and 'MYCN-low' IMR5/75 cells. 
a

RNA

MYCN

H3K $4 \mathrm{me} 3$

H3K4me1

H3K27ac

H3K27me3

H3K $36 \mathrm{me} 3$

H3K9me3

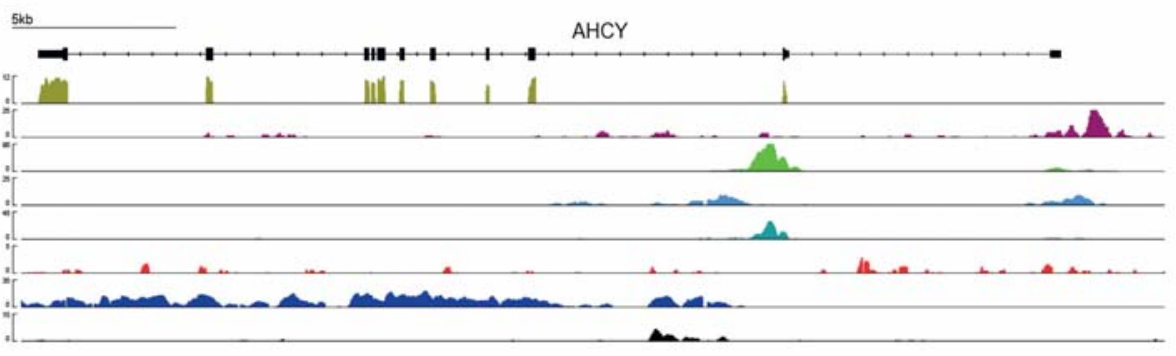

KELLY (MYCN-amplified)

b

RNA

H3K4me3

H3K4me1

H3K27ac

H3K27me3

H3K $36 \mathrm{me} 3$

H3K9me3

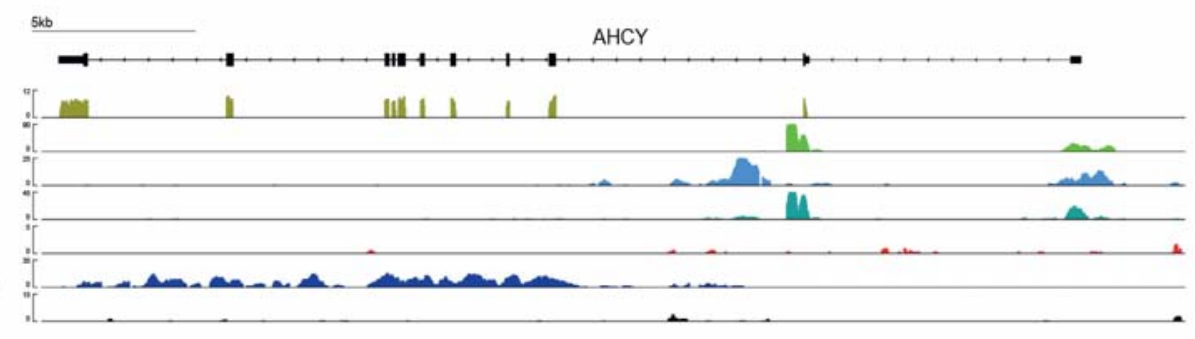

SK-N-FI (MYCN single-copy, no MYCN/MYC expression)

Extended data figure 6

a, b, RNA-seq normalized reads and input normalized read counts of ChIP-seq experiments for histone modifications and MYCN at the AHCY gene locus in MYCN-amplified KELLY cells (a) and in MYCN single-copy SK-N-FI cells (b) lacking MYC(N) expression. 
a

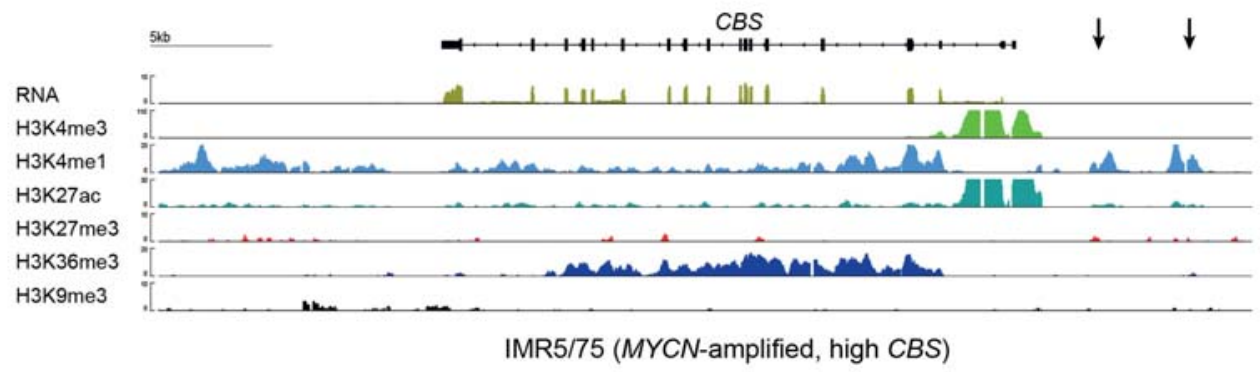

b

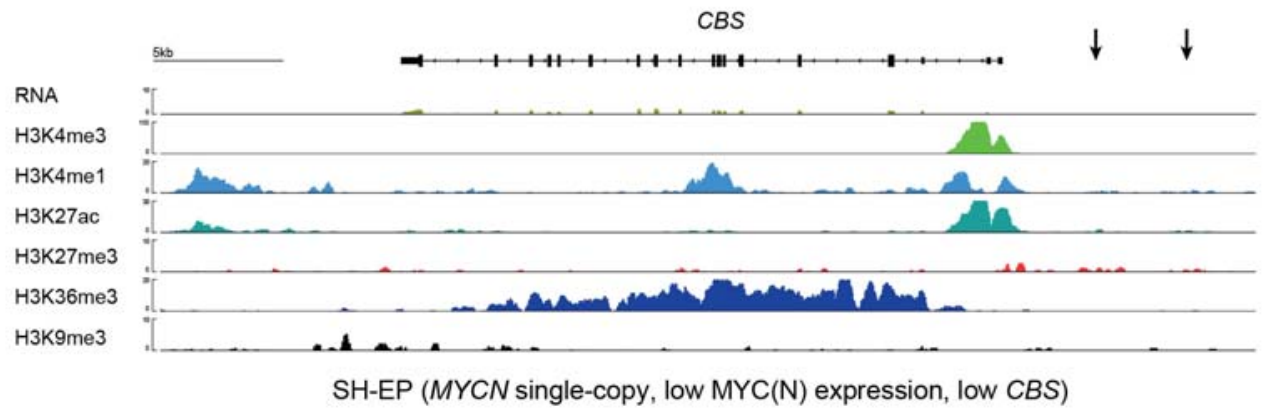

C

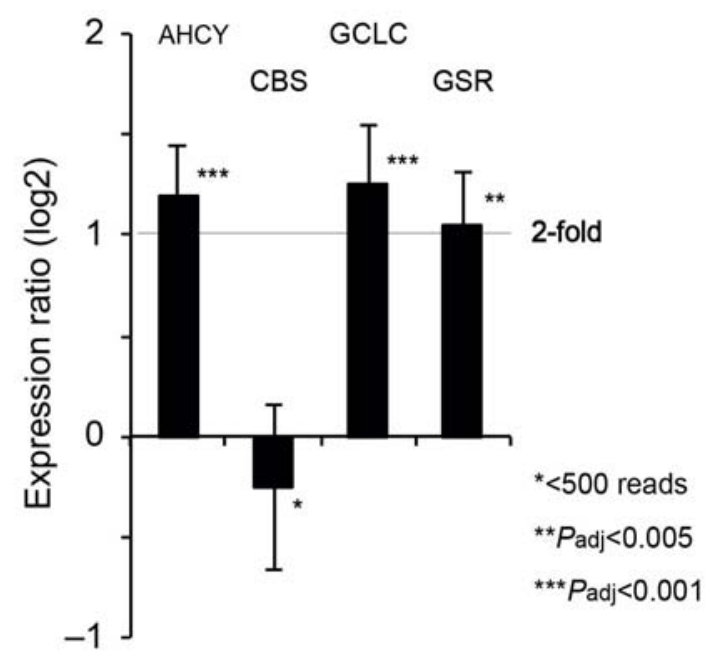

\section{Tet21N (SHEP-MYCNt)}

MYCN high vs low

\section{Extended data figure 7}

a, b, RNA-seq normalized reads and input normalized read counts of ChIP-seq experiments for histone modifications at the CBS gene locus in MYCN-amplified IMR5/75 cells (a) with high CBS expression and active transsulfuration and MYCN single-copy SH-EP cells (b) with low CBS expression and inactive transsulfuration.

c, relative mRNA expression in Tet21N (SH-EP-MYCNt) cells harboring an inducible MYCN transgene upon MYCN induction. Tet21N and parental SH-EP cells have low CBS expression and inactive transsulfuration. MYCN induction has a significant effect on AHCY, GCLC and GSR but not on CBS expression; means \pm SD. 


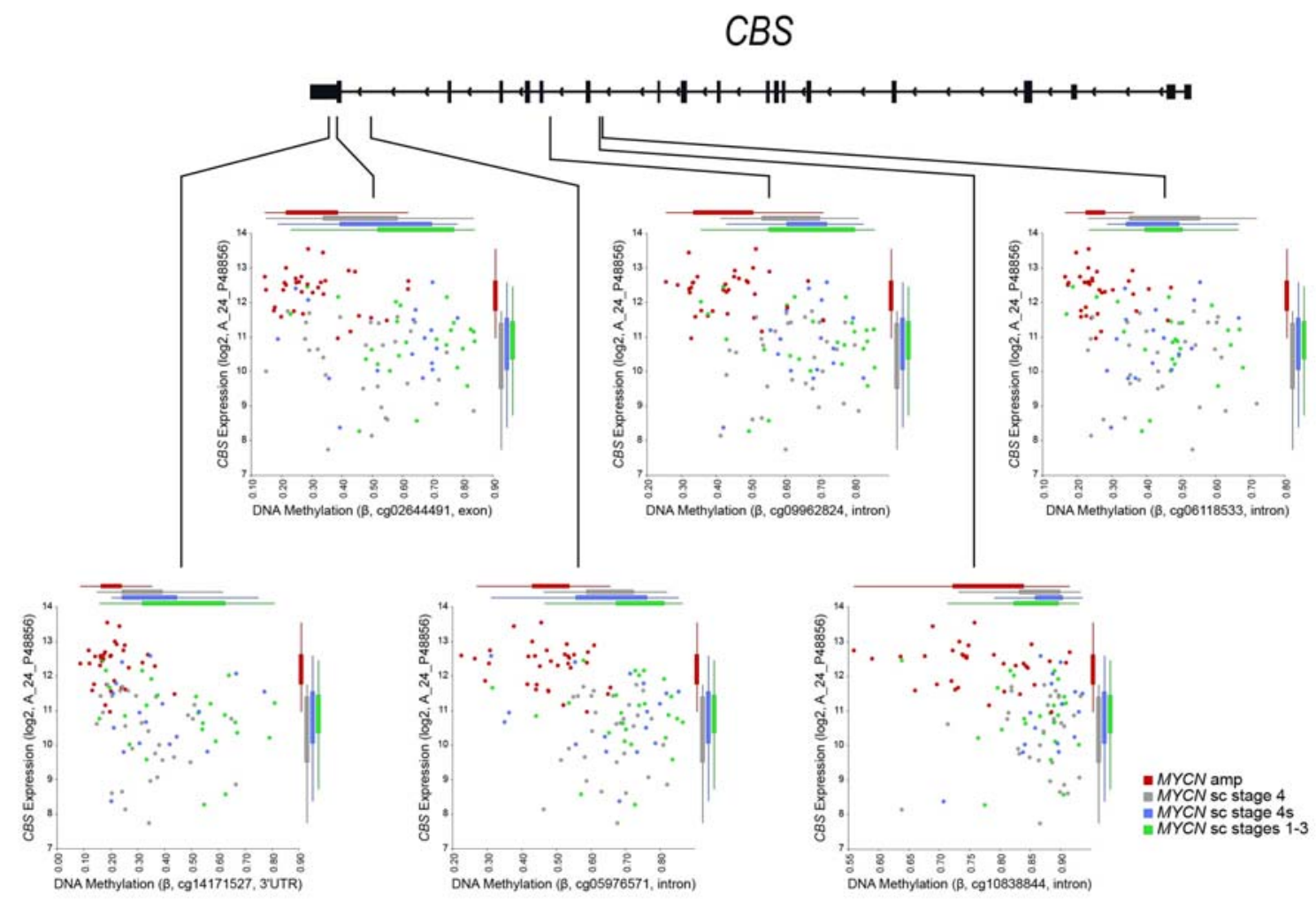

\section{Extended data figure 8}

Genomic position of CBS-annotated CpGs whose methylation is significantly associated with CBS expression and patient risk ( $p<0.01$, Wilcoxon rank-sum statistics and $p<0.05$, Fisher exact test). DNA methylation assessed by Infinium HumanMethylation450 BeadChips and CBS expression assessed by $44 \mathrm{k}$ customized Agilent oligonucleotide microarrays in 105 primary neuroblastomas (GEO accession GSE73518, Henrich et al. 2016) are depicted. For all CBS CpGs whose methylation significantly correlated with $C B S$ expression and patient risk, hypomethylation was associated with CBS upregulation and high-risk disease. R2 Genomics Analysis and Visualization Platform (http://r2.amc.nl) was used for data visualization.

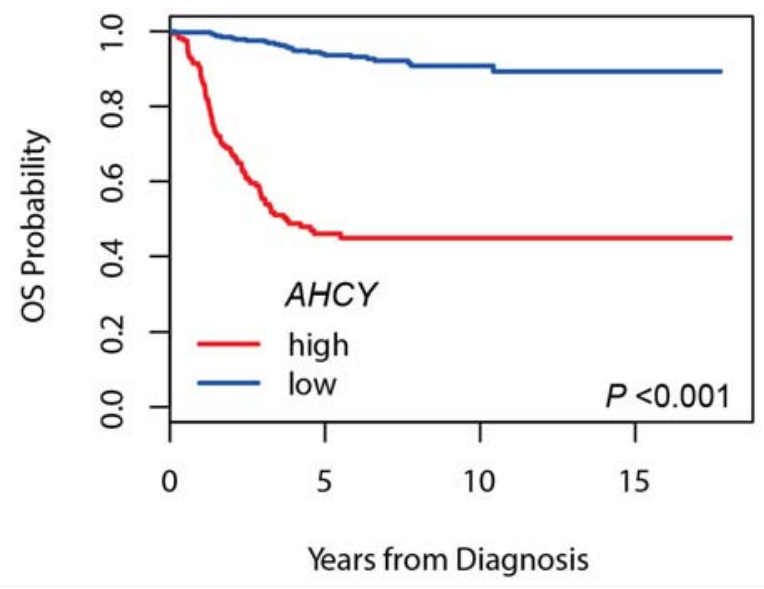

Extended data figure 9

Kaplan-Meier overall survival estimates for subgroups defined by $A H C Y$ expression. The cutoff values for dichotomization of $A H C Y$ expression was estimated by maximally selected log-rank statistics, high $A H C Y$ expression ( $\mathrm{n}=165)$, low $A H C Y$ expression $(\mathrm{n}=333)$. 


\section{Acknowledgements}

This work was supported by the DKFZ-BayerHealthCare Alliance (L.M.B., D.B., S.G., E.M.H. and F.W.), the e:Med initiative (SYSMED-NB, grant no. 01ZX1307D to F.W., T.H. and M. S.), the German Cancer Consortium (DKTK) Joint Funding program, the BMBF MYC-NET grant no. 0316076A to F.W. and T.H., the ERACoSysMed grant Optimize-NB to T.H. and F.W., the European Union grant no. 259348 to F.W., the German Cancer Research Center (DKFZ) intramural program for interaction projects and the DKFZ-Heidelberg Center for Personalized Oncology (HIPO) \& National Center for Tumor Diseases (NCT) Precision Oncology Program (F.W.) and the Berlin Institute of Health (M. S.). S.W. was funded by BfR FKZ1329-468. M.G. and F.P. were supported by PhD fellowships of the German-Israeli Helmholtz Research School in Cancer Biology. The group of J. R. is supported by Merck KGaA. A.F.F thanks Julia Lochead and Tatjana Ryl for experimental support and Carina Thomé for help with RTCA measurements. We are indebted to the patients and their parents of making available the tumor specimens that were analyzed within this study, and we thank the German neuroblastoma biobank for providing patient samples. The Institutional Review Board (IRB) approved collection and use of all specimens in this study. We would like to thank FrankDetlef Scholle and Sebastian Räse (Bayer AG) for automatic microscopic image analysis (MYCN SL screen), our colleagues Young-Gyu Park and Denise Brünig for technical assistance, and Kathy Astrahantseff, Tobias Dick, Maria Llamazares and Theodor C. H. Cole for critical reading of the manuscript. We thank the Metabolomics Core Technology Platform at the University of Heidelberg and Rüdiger Hell for providing data of amino acids and other metabolites. We would also like to acknowledge the DKFZ core facility, helping us to obtain high-quality data of sequencing and DNA methylation analyses.

\section{Contributions}

The study was conceived by A.F.F, H.A and F.W. Metabolic assays and viability studies were conducted and analyzed by H.A., A.F.F., L.M.B., M.S., E.M.H., J.K. and S.G.. S.G. performed the MYCN-synthetic lethal siRNA screen. M.G. helped with MYCN-synthetic lethal screen optimization. C.S., S.S., A.F.F and C.H. assisted with bioinformatic analyses. M.G., F.P., S.H., and D.D. performed and analyzed ChIP-seq and ChIPmentation experiments. M.N.-H., M.Z. and M.S. provided tumor proteomic data. E.B., M.G., E.M.H. and J.K. conducted RNA-seq experiments. Regulable cell models were established and colony formation assays performed by J.K.. Western blotting was conducted by S.G., E.M.H. and J.K.. G.P. and M.B. implemented and analyzed LC-MS measurements. K.-O.H. contributed DNA methylation analyses. B.N., C.S., J.H.R., M.F., I.A., S.W.. contributed reagents, 
materials and analysis tools. The manuscript was prepared by F.W., T.H., H.A., A.F.F., L.M.B. and S.G.. All authors approved the current version of the manuscript.

\section{Material and Methods}

\section{Experimental in vitro procedures}

\section{Cell Culture}

Human neuroblastoma (IMR5/75, KELLY, SiMa, NBL-S, SK-N-FI) and NCI-H23 cells (human NSCLC) were cultivated at $37^{\circ} \mathrm{C}$ with $5 \% \mathrm{CO}_{2}$ in RPMI 1640 medium (Gibco, Thermo Fisher Scientific, California, USA) supplemented with $10 \%$ fetal calf serum (FCS; Gibco), and penicillin/streptomycin (AppliChem, Darmstadt, Germany). Tunable cell lines, IMR5/75 MYCN shRNA, SH-EP MYCN transgene $($ Tet21N) and NCl-H23 MYC shRNA were generated and cultured as described previously ${ }^{14,20}$. Cell line identity/unique SNP profiles were confirmed by the Multiplexion Multiplex Cell Authentication service (Heidelberg, Germany) as described recently ${ }^{41}$. To assess the effects of amino acid deprivation, cells were cultivated using modified amino acid-free DMEM powder (PAN-Biotech, Aidenbach, Germany) supplemented with individual amino acids (Sigma-Aldrich, Munich, Germany) as indicated, at final concentrations used in standard DMEM.

\section{Analysis of Cell Viability and Proliferation}

The impact of various treatments on cellular proliferation/viability was assessed using an SRB assay as described previously ${ }^{42}$. To determine changes in cellular proliferation, approximately $2 \times 10^{4}$ cells were seeded per well (48-well format) in full medium. After $24 \mathrm{~h}$, cells were washed with PBS, fed with the chosen medium and treated as indicated. Cell viability was analyzed in full or cystine $\left(\mathrm{Cys}_{2}\right)$-free

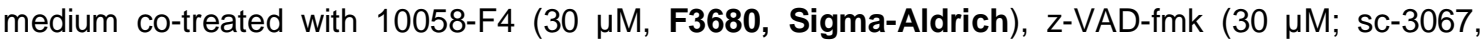
Santa Cruz), bafilomycin A1 (200 nM; sc-201550, Santa Cruz), necrostatin-1 (20 $\mu$ M; N9037, SigmaAldrich), ferrostatin-1(fer-1; $5 \mu \mathrm{M}$, SML0583, Sigma Aldrich), Trolox (100 $\mu \mathrm{M}$; 238813; Sigma-Aldrich), or ciclopirox olamine (CPX; $1 \mu \mathrm{M}$, sc-204688, Santa Cruz). Cells were fixed with ice-cold 10\% trichloroacetic acid (TCA) for $1 \mathrm{~h}$, stained with $0.054 \% \mathrm{w} / \mathrm{v}$ SRB for $30 \mathrm{~min}$, and absorbance measured at $535 \mathrm{~nm}$ using a Tecan Ultra plate reader (Tecan, Maennedorf, Switzerland) at indicated time points. DNA content analysis was performed by fixing cells with $4 \%$ PFA and staining with FxCycle Violet Stain (Thermo Fisher Scientific) followed by FACS using a MACSQuant Flow Cytometer (Miltenyi Biotec, Bergisch Gladbach, Germany).

Doubling times were calculated using two different methods: (i) Impedance measurements were assessed using the RTCA system (Roche) by seeding at different cell densities and registering impedance signals every 20 min. (ii) Standard growth curves were generated by counting cells by FACS (MACSQuant Flow Cytometer) at indicated time points and excluding propidium iodide (PI)positive cells as necrotic. In both methods, exponential curves were fitted and doubling times calculated.

As another measure of cell viability, CellTiter-Blue cell assays were performed in 96-well plates following the manufacturer's instructions (Promega, Mannheim, Germany) using a FLUOstar Optima microplate fluorescence reader (BMG LABTECH, Ortenberg, Germany). $24 \mathrm{~h}$ after seeding, cells were treated and simultaneously induced with $1 \mu \mathrm{g} / \mathrm{ml}$ doxycycline (Dox) (195044, MP Biomedicals, LLC, Illkirch, France) as appropriate. D9 (synthesized and provided by Bayer Pharma AG, Berlin, Germany), erastin (Cay-17754, Biomol GmbH, Hamburg, Germany), RSL-3 (200 nM; Medchemexpress Europe, Sollentuna, Sweden), SAS (599-79-1, Sigma-Aldrich), DL-Propargylglycine (PPG $1 \mathrm{mM}$; P7888, Sigma-Aldrich), fer-1 and Fe (VENOFER, $20 \mathrm{mg} / \mathrm{ml}$, Aca Müller/Adag Pharma AG, Gottmadingen, Germany) were used as indicated, and viability determined $72 \mathrm{~h}$ after treatment/induction. To assess the effects of $\mathrm{Cys}_{2}$ deprivation on cell viability, cells were washed and 
fed with Cys 2 -free DMEM (Gibco) supplemented with $10 \%$ dialyzed FCS (Gibco), 201.3 $\mu$ M Met (6368-3, Sigma-Aldrich) and $4 \mathrm{mM}$ glutamine (Gln; 25030081, Gibco) $48 \mathrm{~h}$ after seeding. To determine cell death rescue potential, $\mathrm{Cys}_{2}$-deprived cells were also co-treated with homocysteine (Hcy; 69453, Sigma-Aldrich), cystathionine (Cysta; C7505, Sigma-Aldrich) or GSH. We further compared effects on cell viability following $\mathrm{Cys}_{2}$ and Gln depletion. Fluorescence was read (540/580nm) $24 \mathrm{~h}$ and $48 \mathrm{~h}$ after deprivation/treatment.

\section{Colony formation assay}

IMR5/75- (5,000 cells/well) and SH-EP-AHCYsh (1,000 cells/well) were seeded in six-well plates and simultaneously treated with doxycycline $(1 \mu \mathrm{g} / \mathrm{ml})$ to induce the $A H C Y$-targeting shRNA. Cells were fixed (11\% glutaraldehyde; Sigma Aldrich) and Giemsa-stained five (SH-EP-AHCYsh) or seven days (IMR5/75-AHCYsh) later. Colony counting was performed using a Gel Doc Documentation System and Quantity One software (Bio-Rad) and quantification using Excel software.

\section{Metabolite profiling and protein analysis}

\section{Metabolite Analysis by Gas Chromatography/Mass Spectrometry (GC/MS) Extraction}

Approximately $6 \times 10^{6}$ cells of MYCN-high and MYCN-low expressing IMR5/75, including 10058-F4(30 $\mathrm{MM}$; F3680; Sigma-Aldrich), or DMSO-treated MYCN-high IMR5/75, were harvested and intracellular GSH and metabolites were measured. Intracellular glutathione levels were further measured as previously described ${ }^{43}$.

Harvested cells were washed twice with $0.9 \%$ ice-cold $\mathrm{NaCl}$ solution and snap-frozen in liquid nitrogen. Frozen pellets were extracted in $180 \mu \mathrm{l}$ of methanol with vigorous shaking for $15 \mathrm{~min}$ at $70^{\circ} \mathrm{C}$. As internal standard, $5 \mu \mathrm{l}$ ribitol $(0.2 \mathrm{mg} / \mathrm{ml}$, A5502, Sigma Aldrich) were added to each sample. Polar and organic phases were separated with $100 \mu \mathrm{l}$ chloroform (shaking samples for $5 \mathrm{~min}$ at $37^{\circ} \mathrm{C}$ ), and $200 \mu \mathrm{l}$ water per sample. Following centrifugation $(11,000 \times \mathrm{g}, 10 \mathrm{~min}), 300 \mu \mathrm{l}$ of the polar (upper) phase were dried in a vacuum concentrator (Eppendorf Concentrator Plus) without heating for derivatization.

\section{Derivatization (Methoximation and Silylation)}

Pellets were re-dissolved in $20 \mu \mathrm{l}$ methoximation reagent containing $20 \mathrm{mg} / \mathrm{ml}$ methoxyamine hydrochloride (226904, Sigma-Aldrich) in pyridine (270970, Sigma-Aldrich) and incubated for $2 \mathrm{~h}$ at $37^{\circ} \mathrm{C}$ with shaking. For silylation, $32.2 \mu \mathrm{l} N$-methyl- $N$-(trimethylsilyl)trifluoroacetamide (MSTFA; M7891, Sigma-Aldrich) and $2.8 \mu$ l Alkane Standard Mixture $\left(50 \mathrm{mg} / \mathrm{ml} \mathrm{C}_{10}-\mathrm{C}_{40} ; 68281\right.$, Fluka) were added to each sample. After incubation for $30 \mathrm{~min}$ at $37^{\circ} \mathrm{C}$, samples were transferred to glass vials for $\mathrm{GC} / \mathrm{MS}$ analysis.

\section{Gas Chromatography/Mass Spectrometry (GC/MS) Analysis}

A GC/MS-QP2010 Plus (Shimadzu®) fitted with a Zebron ZB 5MS column (Phenomenex®; $30 \mathrm{~m} \times$ $0.25 \mathrm{~mm} \times 0.25 \mu \mathrm{m}$ ) was used for GC/MS analyses. The GC was operated with an injection temperature of $230^{\circ} \mathrm{C}$ and $2 \mu \mathrm{l}$ of each sample were injected with split 10 mode. The GC temperature program started with a $1 \mathrm{~min}$ hold at $70^{\circ} \mathrm{C}$ followed by a $6^{\circ} \mathrm{C} / \mathrm{min}$ ramp to $310^{\circ} \mathrm{C}$, a $20^{\circ} \mathrm{C} / \mathrm{min}$ ramp to $330^{\circ} \mathrm{C}$ and a bake-out for $5 \mathrm{~min}$ at $330^{\circ} \mathrm{C}$ using helium as carrier gas with constant linear velocity. MS was operated with ion source and interface temperatures of $250^{\circ} \mathrm{C}$ and a scan range $(\mathrm{m} / \mathrm{z})$ of $40-1000$ with an event time of $0.3 \mathrm{sec}$. "GCMS solution" software (Shimadzu®) was used for data processing.

\section{Quantification of Amino Acids}

Pellets of $2 \times 10^{6}$ cells were extracted with $0.1 \mathrm{ml}$ ice-cold $0.1 \mathrm{M} \mathrm{HCl}$. Non-thiol-containing amino acids were quantified after specific fluorescent labeling with AccQ-TagTM (Waters, Eschborn, Germany) as described by Yang et $\mathrm{al}^{44}$. Cysteine levels were determined after labeling with monobromobimane (Calbiochem, Merck, Darmstadt, Germany) as described before ${ }^{45}$. 


\section{Western Blot Analysis of Proteins in Cell Extracts}

Whole cell lysates were prepared and protein expression visualized as previously described ${ }^{46}$. Protein lysates (50 $\mu \mathrm{g} / \mathrm{lane}$ ) were separated on $12.5 \%$ SDSPAGE. Blots were probed with antibodies directed against MYCN (\#sc-53993, Santa Cruz, 1:1000), c-MYC (ab32072, Abcam, 1:1000), SAHH (A-11) (AHCY antibody, sc-271389, Santa Cruz, 1:1000) or $\beta$-actin-conjugated (ab20272, Abcam, 1:5000). HSR-peroxidase labeled anti-mouse (115-035-003, Dianova, 1:1000) or anti-rabbit (111-035-144, Dianova, 1:1000) antibodies were used as secondary antibodies. Proteins were visualized using ECL detection reagents (Amersham/GE Healthcare, Freiburg, Germany) and a chemiluminescence reader (VILBER, Eberhardzell, Germany). Protein quantification was performed using ImageJ software (https://imagej.net).

\section{Flow cytometry}

\section{Analysis of Intracellular ROS Level}

Approximately $10^{5}$ cells were seeded in six-well plates. MYCN-low populations were established by incubating cells with $1 \mu \mathrm{g} / \mathrm{ml}$ doxycycline at least $48 \mathrm{~h}$ prior to further treatment. Cells were then fed either with full or cystine-free medium and co-treated with fer-1 $(5 \mu \mathrm{M})$; , liproxstatin-1 $(1 \mu \mathrm{M}$; SML1414; Sigma-Aldrich), CPX (1 $\mu \mathrm{M})$, Trolox $(100 \mu \mathrm{M})$ or glutathione (GSH; $2 \mathrm{mM}$, G4251, SigmaAldrich) for $20 \mathrm{~h}$. Before lipid-ROS being analyzed, medium was removed, C11-BODIPY (581/591) diluted in Hank's Balanced Salt Solution (HBSS; Gibco) and added to wells at a final concentration of $4 \mu \mathrm{M}$. After $15 \mathrm{~min}$ staining at $37^{\circ} \mathrm{C}$ inside the tissue culture incubator, cells were harvested gently and levels of lipid-ROS immediately analyzed using a BD FACS Aria ${ }^{\mathrm{TM}}$ III cell sorter. In addition, total intracellular reactive oxygen species (ROS) levels in $\mathrm{Cys}_{2}$-deprived cells were determined using CellROX® (Thermo Fisher Scientific). Approximately $10^{5}$ cells were seeded in 12-well plates. 'MYCNlow' populations were established by incubating cells with $1 \mu \mathrm{g} / \mathrm{ml}$ doxycycline, at least $48 \mathrm{~h}$ prior to further treatment. To test the effect of MYC inhibition on ROS levels, 'MYC-high' cells were treated with 10058-F4 (30 $\mu \mathrm{M}$ for $24 \mathrm{~h}$ ). Cells were then fed either with full or $\mathrm{Cys}_{2}$-free medium $24 \mathrm{~h}$ before CellROX® was added ( $5 \mu \mathrm{M}$ final concentration). After $30 \mathrm{~min}$ of staining in a cell culture incubator, cells were harvested gently and immediately analyzed using a BD FACS Aria ${ }^{\mathrm{TM}}$ III cell sorter to measure the signal intensity directly proportional to the level of intracellular ROS.

\section{MYCN synthetic lethal screen}

\section{Large-Scale Druggable Genome siRNA Screen}

For high-throughput screening, a Silencer Select siRNA custom library (\#4404034, Ambion) was used encompassing 31,242 unpooled siRNAs targeting 10,414 genes (3 siRNAs per gene), Lipofectamine RNAiMax transfection reagent (Life Technologies) only and On-TARGETplus Non-targeting siRNA \#1 (Dharmacon) served as negative transfection controls, PLK1 (Silencer Select siRNA \#1, Ambion) as positive control. Liquid reverse transfection was performed in 384-well plates using a Freedom EVO 200 robotic platform with a 384 MultiChannel Arm, 8-channel Liquid Handling Arm, Robotic Manipulator Arm and EVOware Plus software (TECAN) as follows: Lipofectamine RNAiMax and siRNA were diluted in Opti-MEM (Life Technologies) at appropriate concentrations, combined, and incubated for 15-40 min before adding MYCN-amplified IMR5/75 cells stably expressing a doxycycline-regulable MYCN shRNA (2,100 cells/well) using a Multidrop dispenser (Thermo Fisher Scientific). Two treatment conditions were screened in triplicate: (A) culture medium only (IMR5/75 'MYCN-high'), and (B) plus doxycycline to induce the shRNA targeting MYCN (IMR5/75 'MYCN-low'). After seeding cells into the transfection mixture, medium only ('MYCN-high' transfection plates) or doxycycline $(1 \mu \mathrm{g} / \mathrm{ml}$ final concentration, 'MYCN-low' transfection plates) was added using a Tecan Evo 200 robotic platform. $96 \mathrm{~h}$ after transfection, cells were fixed with $11 \%$ glutaraldehyde and subsequently Hoechst-stained (10 mg/ml stock in 1× PBS, 1:2500; Invitrogen) using a Tecan Evo 200 robotic platform. The number of Hoechst-positive cell nuclei was determined using an OPERA 
fluorescence microscope based on nine sites per well and a BHC in-house program. We applied Redundant siRNA Activity (RSA) to the ratio between MYCN high and MYCN low data to select top ranked hits ${ }^{47}$ and a false discovery rate of $(F D R)$ of 0.2 . Raw and processed data of the druggable whole-genome screen will be made available after publication.

\section{Transcript profiling tumors and cell lines}

\section{RNA-sequencing (RNA-seq)}

Total RNA was isolated using the miRNeasy Mini Kit (Qiagen) and depleted from ribosomal RNAs using the Ribo-Zero rRNA Removal Kit (Illumina) according to the manufacturers' protocols. RNA libraries were prepared using the NEBNext Ultra Directional RNA Library Prep Kit for Illumina (New England BioLabs) with following changes to the manufacturer's instructions: RNA was fragmented for $20 \mathrm{~min}$ at $94^{\circ} \mathrm{C}$, followed by first strand cDNA synthesis for $10 \mathrm{~min}$ at $25^{\circ} \mathrm{C}, 50 \mathrm{~min}$ at $42^{\circ} \mathrm{C}$ and $15 \mathrm{~min}$ at $70^{\circ} \mathrm{C}$. Adapter-ligated DNA was size-selected with a bead:DNA ratio of 0.4 (AMPure XP beads, Beckman Coulter), removing index primer and short fragments. Quality, quantity and sizing (approximately $320 \mathrm{bp}$ ) of the RNA library were checked using a DNA High Sensitivity DNA chip run on a 2100 Bioanalyzer sequencing platform (German Cancer Research Center Core facility).

\section{Data analysis RNA-seq}

RNA-seq expression profiles from 498 primary neuroblastomas ${ }^{48}$ (GSE62564) were analyzed. The Wilcoxon rank sum test was used to test an association between candidate gene expression and amplified MYCN oncogene. Maximally selected log-rank statistics were used to describe the relationship between patient survival and candidate gene expression, and resulting expression cut points were used for dichotomization. Survival curves were estimated using the Kaplan-Meier method. Cox regression was used to investigate the prognostic power of candidate gene expression adjusting for established prognostic variables. Parameter estimate shrinkage was applied to correct for potential overestimation of the hazard ratio estimate due to cut point selection ${ }^{49}$. Pearson correlation coefficients were calculated to estimate linear dependence between expression values of candidate genes.

Raw RNA-seq sequences neuroblastoma cell lines and model systems were mapped to the UCSC hg19 genome by STAR ${ }^{50}$ with default parameters. The corresponding bigwig files of each sample were calculated by RSeQC ${ }^{51}$ and normalized to the wigsum of 100000000 . RNA-seq sequences from the IMR5/75 MYCN-high and MYCN-low cells were processed as previously described ${ }^{32}$.

\section{Epigenetic characterization of tumors and cell lines}

\section{Chromatin immunoprecipitation DNA-sequencing (ChIP-seq) analysis of histone modifications in neuroblastoma primary tumors and cell lines}

Formaldehyde cross-linking of cells, cell lysis, sonication, chromatin immunoprecipitation (IP) and library preparation were performed as described previously ${ }^{52}$, starting with approximately $4 \times 10^{6}$ cells $\left(1 \times 10^{6}\right.$ cells per individual IP). Direct cell lysis for each sample was achieved by $30 \mathrm{~min}$ incubation on ice in $950 \mu \mathrm{L}$ RIPA I (10 mM Tris-HCl pH 8.0, $1 \mathrm{mM}$ EDTA pH 8.0, $140 \mathrm{mM} \mathrm{NaCl}, 0.2 \%$ SDS, $0.1 \%$ $\mathrm{DOC})$. Tissue disruption, formaldehyde fixation and sonication of tumor material was done as previously published ${ }^{53}$, using approximately $30 \mathrm{mg}$ of fresh-frozen tumor tissue per individual ChIPseq experiment. All subsequent steps were performed analogous to cell line experiments. A Bioruptor Plus sonication device (Diagenode) was used for high intensity sonication for 30-60 min each with 30s on and 30s off intervals. Following antibodies were used for IP: H3K4me3 (ab8580, Abcam), H3K4me1 (ab8895, Abcam), H3K27Ac (ab4729, Abcam), H3K27me3 (39155, Active Motif), H3K36me3 (ab9050, Abcam), H3K9me3 (ab8898, Abcam). Library preparation was performed using the NEBNext Ultra DNA Library Prep Kit (New England Biolabs) according to the manufacturer's protocol. Samples were mixed in equal molar ratios and sequenced on an Illumina sequencing platform. 


\section{ChIPmentation of MYCN transcription factor in neuroblastoma cell lines}

Formaldehyde cross-linking, cell lysis, sonication and chromatin immunoprecipitation were performed as previously described ${ }^{54}$, adding the Chipmentation module by Schmidl et al. ${ }^{55}$ with following changes: a Bioruptor Plus with automated cooling $\left(4^{\circ} \mathrm{C}\right)$ was used for high intensity sonication (20-30 min each with $30 \mathrm{sec}$ on and $30 \mathrm{sec}$ off intervals), and $10 \mu \mathrm{g}$ MYCN antibody (MYCN (sc-53993, Santa Cruz) and $10^{6}$ cells for chromatin immunoprecipitation. The tagmentation reaction (Illumina Nextera DNA library Prep Kit) was performed at $37^{\circ} \mathrm{C}$ for 1 min with the bead-bound chromatin sample or $5 \mathrm{ng}$ purified input DNA for normalization. After de-crosslinking, purified samples were amplified using dual index barcodes of the Nextera Index kit and 13 PCR cycles. Enriched libraries were purified and pooled. ChIPmentation libraries were sequenced (50 bases single-end) on the Illumina sequencing platform (German Cancer Research Center Core facility).

\section{Data analysis ChIP-seq and ChIPmentation}

Single end reads were aligned to the hg19 genome using Bowtie2 (version 2.1.0), keeping uniquely aligned reads only. BAM-Files of aligned reads were further processed using the deepTools suite ${ }^{56}$. Input files were subtracted from treatment files using the bamCompare tool, applying the SES method for normalization of signal to noise. Resulting signals were normalized to an average $1 \mathrm{X}$ coverage to produce signal (bigWig) files. Peaks were called using the MACS 1.4 tool using default parameters. Data sets from cell lines and primary tumors will be made available after publication.

\section{DNA methylation analysis}

DNA methylation and gene expression data from 105 primary neuroblastomas assessed by Infinium HumanMethylation450 BeadChips and 44k Agilent oligonucleotide microarrays ${ }^{13}$ (GEO accession GSE73518) were analyzed for candidate loci. For investigating correlation between DNA methylation and expression, maximally selected Wilcoxon rank-sum statistics were used estimating CpG methylation cut points that separate patient groups with differential gene expression. To test the null hypothesis that odds ratios estimating the association of methylation-separated patient subgroups with high-risk disease were 1, a Fisher exact test was used. R2 Genomics Analysis and Visualization Platform (http://r2.amc.nl) was used to visualize expression/methylation of selected gene/CpG pairs.

\section{Tumor proteome analysis}

Patient samples were lysed with $2 \%$ SDS, 50mM ammonium bicarbonate buffer and complete, mini, EDTA-free Protease Inhibitor Cocktail. Samples were homogenized at RT $15 \mathrm{sec}$ for 7 cycles with a 5 sec pause using FastPrep-24 ${ }^{\mathrm{TM}} 5 \mathrm{G}$ Homogenizer and heated to $95^{\circ} \mathrm{C}$ for $5 \mathrm{~min}$. Freeze-thaw cycles were repeated seven times. Benzonase was added to each sample and the lysate was clarified by centrifugation at $16 \mathrm{rcf}$ for $30 \mathrm{~min}$ at $4^{\circ} \mathrm{C}$. Protein concentration was then measured using the Bio-Rad DC Protein assay. From each sample, $50 \mu \mathrm{g}$ of protein were taken in duplicate. Proteins were reduced with $10 \mathrm{mM}$ DTT for $30 \mathrm{~min}$ and alkylated with $55 \mathrm{mM}$ iodoacetamide for $30 \mathrm{~min}$. Wessel-Flügge precipitation was performed as previously described ${ }^{57}$. The retrieved protein pellet was resuspended in $6 \mathrm{M}$ Urea, $2 \mathrm{M}$ Thiourea in $10 \mathrm{mM}$ HEPES (pH 8). Proteins were then digested with Lys $\mathrm{C}$ for $2 \mathrm{~h}$; samples were diluted in $50 \mathrm{mM}$ ammonium bicarbonate buffer to reach less than $2 \mathrm{M}$ urea before trypsin was added for overnight digestion. The peptide solution was acidified and fractionated by SCX on StageTips as described previously ${ }^{58}$. Samples were then separated by reversed phase HPLC on a $2,000 \mathrm{~mm}$ monolithic column and analyzed online by high resolution mass spectrometry on a $Q$ Exactive plus mass instrument as described previously ${ }^{59}$. The resulting raw files were analyzed using MaxQuant software version 1.5.5.160. Default settings were used with 'match between runs' function. Data was searched against a Human Uniprot database (2014-10) and common contaminants at a false discovery rate of $1 \%$ at both the peptide and protein level. The resulting text files were filtered to exclude reverse database hits, potential contaminants, and proteins only identified by site. Plotting and statistics were done using Perseus software version 1.5.5.3. ${ }^{61}$. 
bioRxiv preprint doi: https://doi.org/10.1101/2021.08.10.455675; this version posted August 10, 2021. The copyright holder for this preprint (which was not certified by peer review) is the author/funder, who has granted bioRxiv a license to display the preprint in perpetuity. It is made available under aCC-BY-NC-ND 4.0 International license. 


\section{References}

1 Lin, C. Y. et al. Transcriptional amplification in tumor cells with elevated c-Myc. Cell 151, 56-67, doi:10.1016/j.cell.2012.08.026 (2012).

2 Nie, Z. et al. c-Myc is a universal amplifier of expressed genes in lymphocytes and embryonic stem cells. Cell 151, 68-79, doi:10.1016/j.cell.2012.08.033 (2012).

3 Sabo, A. et al. Selective transcriptional regulation by Myc in cellular growth control and lymphomagenesis. Nature 511, 488-492, doi:10.1038/nature13537 (2014).

$4 \quad$ Walz, S. et al. Activation and repression by oncogenic MYC shape tumour-specific gene expression profiles. Nature 511, 483-487, doi:10.1038/nature13473 (2014).

5 Murphy, D. J. et al. Distinct thresholds govern Myc's biological output in vivo. Cancer cell 14, 447-457, doi:10.1016/j.ccr.2008.10.018 (2008).

6 Maris, J. M. Recent advances in neuroblastoma. The New England journal of medicine 362, 2202-2211, doi:10.1056/NEJMra0804577 (2010).

7 Schwab, M. et al. Amplified DNA with limited homology to myc cellular oncogene is shared by human neuroblastoma cell lines and a neuroblastoma tumour. Nature 305, 245-248 (1983).

8 Brodeur, G. M., Seeger, R. C., Schwab, M., Varmus, H. E. \& Bishop, J. M. Amplification of $\mathrm{N}$-myc in untreated human neuroblastomas correlates with advanced disease stage. Science 224, 1121-1124 (1984).

9 Brodeur, G. M. \& Bagatell, R. Mechanisms of neuroblastoma regression. Nature reviews. Clinical oncology 11, 704-713, doi:10.1038/nrclinonc.2014.168 (2014).

10 Westermann, F. et al. Distinct transcriptional MYCN/c-MYC activities are associated with spontaneous regression or malignant progression in neuroblastomas. Genome biology 9, R150, doi:10.1186/gb-2008-9-10-r150 (2008).

11 Teitz, T. et al. Caspase 8 is deleted or silenced preferentially in childhood neuroblastomas with amplification of MYCN. Nature medicine 6, 529-535, doi:10.1038/75007 (2000).

12 Van Maerken, T. et al. Small-molecule MDM2 antagonists as a new therapy concept for neuroblastoma. Cancer research 66, 9646-9655, doi:10.1158/0008-5472.CAN-060792 (2006).

13 Henrich, K. O. et al. Integrative Genome-Scale Analysis Identifies Epigenetic Mechanisms of Transcriptional Deregulation in Unfavorable Neuroblastomas. Cancer research 76, 5523-5537, doi:10.1158/0008-5472.CAN-15-2507 (2016).

14 Muth, D. et al. Transcriptional repression of SKP2 is impaired in MYCN-amplified neuroblastoma. Cancer research 70, 3791-3802, doi:10.1158/0008-5472.CAN-091245 (2010).

15 Zirath, H. et al. MYC inhibition induces metabolic changes leading to accumulation of lipid droplets in tumor cells. Proceedings of the National Academy of Sciences of the United States of America 110, 10258-10263, doi:10.1073/pnas.1222404110 (2013).

16 Qing, G. et al. ATF4 regulates MYC-mediated neuroblastoma cell death upon glutamine deprivation. Cancer cell 22, 631-644, doi:10.1016/j.ccr.2012.09.021 (2012).

17 Wise, D. R. et al. Myc regulates a transcriptional program that stimulates mitochondrial glutaminolysis and leads to glutamine addiction. Proceedings of the National Academy of Sciences of the United States of America 105, 18782-18787, doi:10.1073/pnas.0810199105 (2008).

18 Yuneva, M., Zamboni, N., Oefner, P., Sachidanandam, R. \& Lazebnik, Y. Deficiency in glutamine but not glucose induces MYC-dependent apoptosis in human cells. $J$ Cell Biol 178, 93-105, doi:10.1083/jcb.200703099 (2007).

19 Gao, P. et al. c-Myc suppression of $\mathrm{miR}-23 \mathrm{a} / \mathrm{b}$ enhances mitochondrial glutaminase expression and glutamine metabolism. Nature 458, 762-765, doi:10.1038/nature07823 (2009).

20 Lutz, W. et al. Conditional expression of N-myc in human neuroblastoma cells increases expression of alpha-prothymosin and ornithine decarboxylase and accelerates progression into S-phase early after mitogenic stimulation of quiescent cells. Oncogene 13, 803-812 (1996). 
21 Dixon, S. J. et al. Ferroptosis: an iron-dependent form of nonapoptotic cell death. Cell 149, 1060-1072, doi:10.1016/j.cell.2012.03.042 (2012).

22 Yang, W. S. et al. Regulation of ferroptotic cancer cell death by GPX4. Cell 156, 317331, doi:10.1016/j.cell.2013.12.010 (2014).

23 Gao, M., Monian, P., Quadri, N., Ramasamy, R. \& Jiang, X. Glutaminolysis and Transferrin Regulate Ferroptosis. Mol Cell 59, 298-308, doi:10.1016/j.molcel.2015.06.011 (2015).

24 Lu, S. C. Regulation of glutathione synthesis. Molecular aspects of medicine 30, 4259, doi:10.1016/j.mam.2008.05.005 (2009).

25 O'Donnell, K. A. et al. Activation of transferrin receptor 1 by c-Myc enhances cellular proliferation and tumorigenesis. Molecular and cellular biology 26, 2373-2386, doi:10.1128/MCB.26.6.2373-2386.2006 (2006).

26 McBean, G. J. The transsulfuration pathway: a source of cysteine for glutathione in astrocytes. Amino acids 42, 199-205, doi:10.1007/s00726-011-0864-8 (2012).

27 Bannai, S. \& Kitamura, E. Transport interaction of L-cystine and L-glutamate in human diploid fibroblasts in culture. J Biol Chem 255, 2372-2376 (1980).

28 Dann, S. G. et al. Reciprocal regulation of amino acid import and epigenetic state through Lat1 and EZH2. The EMBO journal 34, 1773-1785, doi:10.15252/embj.201488166 (2015).

29 Yagoda, N. et al. RAS-RAF-MEK-dependent oxidative cell death involving voltagedependent anion channels. Nature 447, 864-868, doi:10.1038/nature05859 (2007).

30 Kanai, Y. \& Endou, H. Functional properties of multispecific amino acid transporters and their implications to transporter-mediated toxicity. J Toxicol Sci 28, 1-17 (2003).

31 Jiang, X. et al. Functional Characterization of D9, a Novel Deazaneplanocin A (DZNep) Analog, in Targeting Acute Myeloid Leukemia (AML). PloS one 10, e0122983, doi:10.1371/journal.pone.0122983 (2015).

32 Ryl, T. et al. Newly born cancer cells escape chemotherapy. submitted.

33 Zhang, W. et al. Comparison of RNA-seq and microarray-based models for clinical endpoint prediction. Genome biology 16, 133, doi:10.1186/s13059-015-0694-1 (2015).

34 Vogan, K. et al. Absence of p53 gene mutations in primary neuroblastomas. Cancer research 53, 5269-5273 (1993).

35 Chen, L. et al. p53 is a direct transcriptional target of MYCN in neuroblastoma. Cancer research 70, 1377-1388, doi:10.1158/0008-5472.CAN-09-2598 (2010).

36 Jiang, L. et al. Ferroptosis as a p53-mediated activity during tumour suppression. Nature 520, 57-62, doi:10.1038/nature14344 (2015).

37 Cardona, C. et al. Expression of Gls and Gls2 glutaminase isoforms in astrocytes. Glia 63, 365-382, doi:10.1002/glia.22758 (2015).

38 Cassago, A. et al. Mitochondrial localization and structure-based phosphate activation mechanism of Glutaminase $C$ with implications for cancer metabolism. Proceedings of the National Academy of Sciences of the United States of America 109, 1092-1097, doi:10.1073/pnas.1112495109 (2012).

39 Green, D. R. \& Victor, B. The pantheon of the fallen: why are there so many forms of cell death? Trends in cell biology 22, 555-556, doi:10.1016/j.tcb.2012.08.008 (2012).

40 Cao, J. Y. \& Dixon, S. J. Mechanisms of ferroptosis. Cell Mol Life Sci 73, 2195-2209, doi:10.1007/s00018-016-2194-1 (2016).

41 Castro, F. et al. High-throughput SNP-based authentication of human cell lines. International journal of cancer 132, 308-314, doi:10.1002/ijc.27675 (2013).

42 Vichai, V. \& Kirtikara, K. Sulforhodamine B colorimetric assay for cytotoxicity screening. Nat Protoc 1, 1112-1116, doi:10.1038/nprot.2006.179 (2006).

43 Rahman, I., Kode, A. \& Biswas, S. K. Assay for quantitative determination of glutathione and glutathione disulfide levels using enzymatic recycling method. Nat Protoc 1, 3159-3165, doi:10.1038/nprot.2006.378 (2006).

44 Yang, Y. et al. Relation between chemotaxis and consumption of amino acids in bacteria. Molecular microbiology 96, 1272-1282, doi:10.1111/mmi.13006 (2015). 
45 Wirtz, M., Droux, M. \& Hell, R. O-acetylserine (thiol) lyase: an enigmatic enzyme of plant cysteine biosynthesis revisited in Arabidopsis thaliana. $J$ Exp Bot 55, 17851798, doi:10.1093/jxb/erh201 (2004).

46 Afanasyeva, E. A. et al. MicroRNA miR-885-5p targets CDK2 and MCM5, activates p53 and inhibits proliferation and survival. Cell death and differentiation 18, 974-984, doi:10.1038/cdd.2010.164 (2011).

47 Konig, R. et al. A probability-based approach for the analysis of large-scale RNAi screens. Nature methods 4, 847-849, doi:10.1038/nmeth1089 (2007).

48 Su, Z. et al. An investigation of biomarkers derived from legacy microarray data for their utility in the RNA-seq era. Genome biology 15, 523, doi:10.1186/s13059-0140523-y (2014).

49 Schumacher, M., Holländer, N., Schwarzer, G. \& Sauerbrei, W. in Handbook of statistics in clinical oncology (ed J. Crowley) (Marcel Dekker, 2001).

50 Dobin, A. et al. STAR: ultrafast universal RNA-seq aligner. Bioinformatics 29, 15-21, doi:10.1093/bioinformatics/bts635 (2013).

51 Wang, L., Wang, S. \& Li, W. RSeQC: quality control of RNA-seq experiments. Bioinformatics 28, 2184-2185, doi:10.1093/bioinformatics/bts356 (2012).

52 Blecher-Gonen, R. et al. High-throughput chromatin immunoprecipitation for genomewide mapping of in vivo protein-DNA interactions and epigenomic states. Nature protocols 8, 539-554, doi:10.1038/nprot.2013.023 (2013).

53 Dahl, J. A. \& Collas, P. A rapid micro chromatin immunoprecipitation assay (microChIP). Nature protocols 3, 1032-1045, doi:10.1038/nprot.2008.68 (2008).

54 Peifer, M. et al. Telomerase activation by genomic rearrangements in high-risk neuroblastoma. Nature 526, 700-704, doi:10.1038/nature14980 (2015).

55 Schmidl, C., Rendeiro, A. F., Sheffield, N. C. \& Bock, C. ChIPmentation: fast, robust, low-input ChIP-seq for histones and transcription factors. Nature methods 12, 963965, doi:10.1038/nmeth.3542 (2015).

56 Ramirez, F., Dundar, F., Diehl, S., Gruning, B. A. \& Manke, T. deepTools: a flexible platform for exploring deep-sequencing data. Nucleic acids research 42, W187-191, doi:10.1093/nar/gku365 (2014).

57 Puchades, M., Westman, A., Blennow, K. \& Davidsson, P. Removal of sodium dodecyl sulfate from protein samples prior to matrix-assisted laser desorption/ionization mass spectrometry. Rapid communications in mass spectrometry : RCM 13, 344-349, doi:10.1002/(SICl)10970231(19990315)13:5<344::AID-RCM489>3.0.CO;2-V (1999).

58 Kulak, N. A., Pichler, G., Paron, I., Nagaraj, N. \& Mann, M. Minimal, encapsulated proteomic-sample processing applied to copy-number estimation in eukaryotic cells. Nature methods 11, 319-324, doi:10.1038/nmeth.2834 (2014).

59 McShane, E. et al. Kinetic Analysis of Protein Stability Reveals Age-Dependent Degradation. Cell 167, 803-815 e821, doi:10.1016/j.cell.2016.09.015 (2016).

60 Cox, J. \& Mann, M. MaxQuant enables high peptide identification rates, individualized p.p.b.-range mass accuracies and proteome-wide protein quantification. Nature biotechnology 26, 1367-1372, doi:10.1038/nbt.1511 (2008).

61 Tyanova, S. et al. The Perseus computational platform for comprehensive analysis of (prote)omics data. Nature methods 13, 731-740, doi:10.1038/nmeth.3901 (2016). 